This manuscript has been submitted for publication in Geophysical Journal International.

Please note that, the manuscript has not been peer reviewed yet, hence subsequent versions of this manuscript may have slightly different content. If accepted, the final version of this manuscript will be available via the "Peer reviewed Publication DOI" link on the right-hand side of this webpage. 


\title{
DeepPhasePick: A method for Detecting and Picking Seismic Phases from Local Earthquakes based on highly optimized
}

\section{Convolutional and Recurrent Deep Neural Networks}

\author{
Hugo Soto and Bernd Schurr \\ German Research Centre for Geosciences (GFZ), Potsdam, Germany.E-mail: soto@gfz-potsdam.de
}

1 October 2020

\begin{abstract}
SUMMARY
Seismic phase detection, identification and first-onset picking are basic but essential routines to analyse earthquake data. As both the number of seismic stations, globally and regionally, and the number of experiments greatly increase due to ever greater availability of instrumentation, automated data processing becomes more and more essential. E.g., for modern seismic experiments involving 100 s to even 1,000 s instruments, conventional human analyst-based identification and picking of seismic phases is becoming unfeasible, and the introduction of automatic algorithms mandatory. In this paper, we introduce DeepPhasePick, an automatic two-stage method that detects and picks $\mathrm{P}$ and $\mathrm{S}$ seismic phases from local earthquakes. The method is entirely based on highly optimized deep neural networks, consisting of a first stage that detects the phases using a convolutional neural network, and a second stage that uses two recurrent neural networks to pick both phases. Detection is performed on three-component seismograms. P- and S-picking is then conducted on the vertical and the two-horizontal components, respectively. Systematic hyperparameter optimization was applied to select the best model architectures and to define both the filter applied to preprocess the seismic data as well as the characteristics of the window sample used to feed the models. We trained DeepPhasePick using seismic records extracted from two sets of manually-picked event waveforms originating from northern Chile ( $\sim 39,000$ records for detection and $\sim 36,000$ records for picking). In dif-
\end{abstract}




\section{H. Soto \& B. Schurr}

ferent tectonic regimes, DeepPhasePick demonstrated the ability to both detect $\mathrm{P}$ and $\mathrm{S}$ phases from local earthquakes with high accuracy, as well as predict P- and S-phase time onsets with an analyst level of precision. DeepPhasePick additionally computes onset uncertainties based on the Monte Carlo Dropout technique as an approximation of Bayesian inference. This information can then further feed an associator algorithm in an earthquake location procedure.

Key words: Computational seismology - Time-series analysis - Body waves - Neural Networks, fuzzy logic - South America

\section{INTRODUCTION}

One of the most fundamental components in any earthquake hypocenter estimation routine is the identification and picking of seismic phases, primarily $\mathrm{P}$ and $\mathrm{S}$ phases from local earthquakes. In the past, this task was commonly performed manually by analysts, who identified each phase arrival based on their training and experience. However, as the available seismic data has rapidly increased over time, the use of automatic phase detection algorithms has become increasingly necessary.

These automatic algorithms encompass detectors which are based on the energy or frequency content of the seismic waveforms such as STA/LTA (e.g., Allen 1978; Baer \& Kradolfer 1987; Earle \& Shearer 1994; Sleeman \& van Eck|1999; Aldersons 2004; Di Stefano et al. 2006; Diehl et al.2009), those based on correlations of template waveforms against continuous seismic data (e.g., Van Trees 1968; Harris 1991; Gibbons \& Ringdal 2006), and detectors based on the representation of seismic data as a linear combination of orthogonal basis waveforms (Scharf \& Friedlander 1994; Harris 1997, 2001).

Phase detectors based on frequency or energy have been used in the past as part of multi-stage automatic earthquake location procedures that allowed the creation of high-quality earthquake catalogs, e.g., for the Northern Chile region (Sippl et al. 2018; Soto et al. 2019). Correlation detectors, such as the matched filter method (Van Trees 1968), rely on the similarity of known template waveforms and have been widely used for detecting repeating earthquakes (e.g., Nadeau \& Johnson 1998; Igarashi et al. 2003; Kato \& Igarashi| 2012; Huang et al. 2017; Folesky et al. 
2018) or searching for missing events (e.g., Shelly et al. 2007; Peng \& Zhao 2009; Ross et al. 2019a) in different tectonic regimes. Subspace detectors, which are based on orthogonal basis waveforms, have been used for identifying earthquakes associated to aftershock sequences and low-frequency tremors (Maceira et al. 2010, Harris \& Dodge 2011).

Despite the fact that energy-based phase detectors do not require strong prior waveform knowledge, an increase in their ability to detect small onsets also implies higher false positive rates. Correlation detectors can achieve very low false positive rates, but they can solely detect similar signals to the ones already present in the selected template waveforms (Harris 1991). Subspace detectors can further extend the range of detected signals, depending on which detection threshold and subspace dimension parameters are used. However, they are not easy to implement efficiently since they require a high computational cost (see e.g., Harris \& Paik 2006).

All the above described methods exploit a priori assumed attributes of the signals such as energy or waveform similarity. Instead, deep learning is a representation-learning method that learns multiple layers of features (a so called "neural network") directly from input data by applying nonlinear transformations sequentially (LeCun et al. 2015; Goodfellow et al.|2016). In the supervised version of deep learning, the goal is to reduce the calculated error (objective function) between the network predictions and the known labels that the network is fed with. This is achieved by adjusting the units (weights) in each layer after backpropagating the gradients of the objective function, computed over the units in the last layer, to the input layer of the network. The network training consists of repeating the whole process until there is no further decrease in error (LeCun et al. 2015; Goodfellow et al. 2016).

Thanks to the enormous increase in computational calculation capacity, deep learning has in recent years shown stunning results in diverse fields such as image recognition (Krizhevsky et al. 2012), speech recognition (Hinton et al. 2012), language translation (Sutskever et al. 2014) and particle Physics (Kaggle challenge 2014).

Convolutional neural networks (CNNs) are a class of deep neural networks specialized for processing grid-like data (Goodfellow et al. 2016). CNNs stand out due to the fact that they are computationally more efficient, easier to train, and have proven to effectively generalize learned 
features in many supervised tasks, ranging from image and document recognition (LeCun et al. 1990, 1998; Simard et al. 2003; Krizhevsky et al. 2012; Taigman et al. 2014) to seismic waves simulation (Moseley et al. 2018), or volcanic ash particles classification (Shoji et al. 2018).

Originally inspired by the properties observed in the primary visual cortex (PVC) of the mammalian brains (Hubel \& Wiesel 1959, 1962), modern CNN architectures are usually formed by several stages of consecutive operations of convolution, non-linear transformation, pooling, and regularization. In the first stage, a filter (kernel) performs local weighted sums (convolutions) through the input data. This linearly activates or detects local features, emulating the behavior observed in the so-called simple cells of the PVC. The resulting feature maps are transformed by applying a non-linear function. A further function then summarizes its statistics in a local scale (pooling). Usually the maximum (max pooling) within a sub-region is computed in this second function, inspired by the function of PVC complex cells (LeCun et al. 2015; Goodfellow et al. 2016). Convolution is highly efficient and effective because it takes advantage of the local connectivity and invariance to location exhibited by meaningful learnable features in natural signals. Firstly, it detects meaningful locally-connected features by using kernels smaller than the input, which allows units in deeper layers to preserve information from the input layer. Secondly, it applies the same filters all over the input, thus permitting the learning of only one common set of parameters instead of many, as well as ensuring that the same learned representations can be found at different locations in the data. In addition to convolution, pooling in neighboring units in a layer can reduce the dimensions of the learned representation and makes it invariant to small perturbations in the input (LeCun et al. 2015; Goodfellow et al. 2016). Furthermore, regularization techniques help the learned features to better generalize to new unseen data, preventing model overfitting. One of the more effective and most frequently applied examples of regularization is Dropout (Srivastava et al.|2014), where noise is introduced in the output features of a layer aiming at inhibiting the network from learning non-meaningful patterns.

Traditional deep neural networks, including CNNs, are limited by having no memory, hence they cannot extract meaningful contextual information from sequentially structured data. Recurrent neural networks (RNNs; Rumelhart et al. 1986) overcome this constraint by simplistically 
mimicking human beings' mechanism for processing external information. They achieve this by implementing an internal loop that iterates over a sequence of data, element by element, while keeping an internal memory state of data they have already processed. The development of the Long-Short Term Memory RNNs (LSTMs) was one of the major breakthroughs in RNN architecture, since it solved the so-called vanishing gradient problem, whereby simple RNNs proved to be incapable of preserving long-term dependent information (Hochreiter \& Schmidhuber 1997). By carrying information to later points in the sequence, LSTMs are capable of retaining patterns, and therefore learning, from very long sequences. LSTM models also make use of internal dropout and recurrent dropout that help prevent overfitting, so improving model performance. Another significant advancement was the invention of Bidirectional RNNs (BRNNs; Schuster \& Paliwal 1997), which make use of two recurrent layers to process sequences in both forward and backward directions. BRNNs can make learning more effective in sequences where both the past and future contexts can provide valuable insights.

In seismology, CNN models have been recently applied for detection (Ross et al. $\mid 2018 \mathrm{a}) \mathrm{b}$; Zhu \& Beroza 2018; Dokht et al. 2019; Woollam et al. 2019) and association (McBrearty et al. 2019) of $\mathrm{P}$ and $\mathrm{S}$ wave arrivals, as well as for earthquake localization (Kriegerowski et al. 2019; Perol et al. 2018; Zhang et al. 2020). RNN-based networks have been used for predicting approximate earthquake times and locations (Panakkat \& Adeli 2009), and for seismic phase association (Ross et al. 2019b).

The present work adds to these previous studies, and introduces DeepPhasePick, a new automatized two-stage method for detecting and picking seismic $\mathrm{P}$ and $\mathrm{S}$ phases from local earthquakes, entirely based on highly optimized deep neural networks. The first stage in DeepPhasePick consists of an adaptive CNN architecture trained for detecting the phases. Here the type of data preprocessing, as well as the length and position of the seismic phase windows used to train the network, were included among the optimizable hyperparameters. Phase picking is conducted in the second stage by applying two additional adaptive Bidirectional LSTM (BLSTM) networks, which were trained specifically to predict P- and S-phase time onsets. The onsets, and their uncertainties, are 
H. Soto \& B. Schurr

determined on time windows defined based on the predicted probabilities of the $\mathrm{P}$ and $\mathrm{S}$ phases in the detection stage.

Here we demonstrate how the optimized CNN network in the first stage of DeepPhasePick, trained on a rather small dataset of labeled phases in comparison to previous studies, is able to detect with high accuracy $\mathrm{P}$ and $\mathrm{S}$ phases from local earthquakes of different tectonic regimes. We also show how, by leveraging the information of the detected seismic phases, the optimized BLSTM models trained for picking are able to predict P- and S-phase time onsets with analyst levels of precision, while also avoiding inherent human bias.

\section{DATA AND METHODS}

\subsection{Earthquake catalog datasets}

In this study, we used two sets of manually picked event waveforms (Fig. 11). The first set (S1) consists of 1,125 events from the time period between 1996-12-02T00:54:33.89 and 199711-20T02:24:59.41 (Schurr et al.|2006). The second set (S2) contains 1,196 events which occurred from 2007-06-14T01:18:52.24 to 2007-12-13T07:23:39.24 in the area of the 2007 M7.7 Tocopilla earthquake (Schurr et al. 2012). S1 contains mostly plate interface events, whereas S2 contains mostly intermediate-depth intraplate events.

From these earthquake catalogs, we extracted three-component seismograms which we subdivided into three window classes: 25,647 P-phase, 25,647 Noise (N), and 14,397 S-phase windows. $16,234 \mathrm{P}, 16,234 \mathrm{~N}$ and 8,061 $\mathrm{S}$ of these samples were obtained from $\mathrm{S} 1$, and 9,413 P, 9,413 N and $6,336 \mathrm{~S}$ samples from $\mathrm{S} 2$. We used these seismic windows as input data to train adaptive neural networks in phase detection and phase picking tasks, as will be described in the next sections.

\subsection{Hyperparameter optimization of adaptive neural networks}

The architecture of a neural network is defined by its hyperparameters, such as the number of layers in the network, the training learning rate, and the batch size used during training. The selection of the model hyperparameters is key when implementing a supervised deep learning task, 


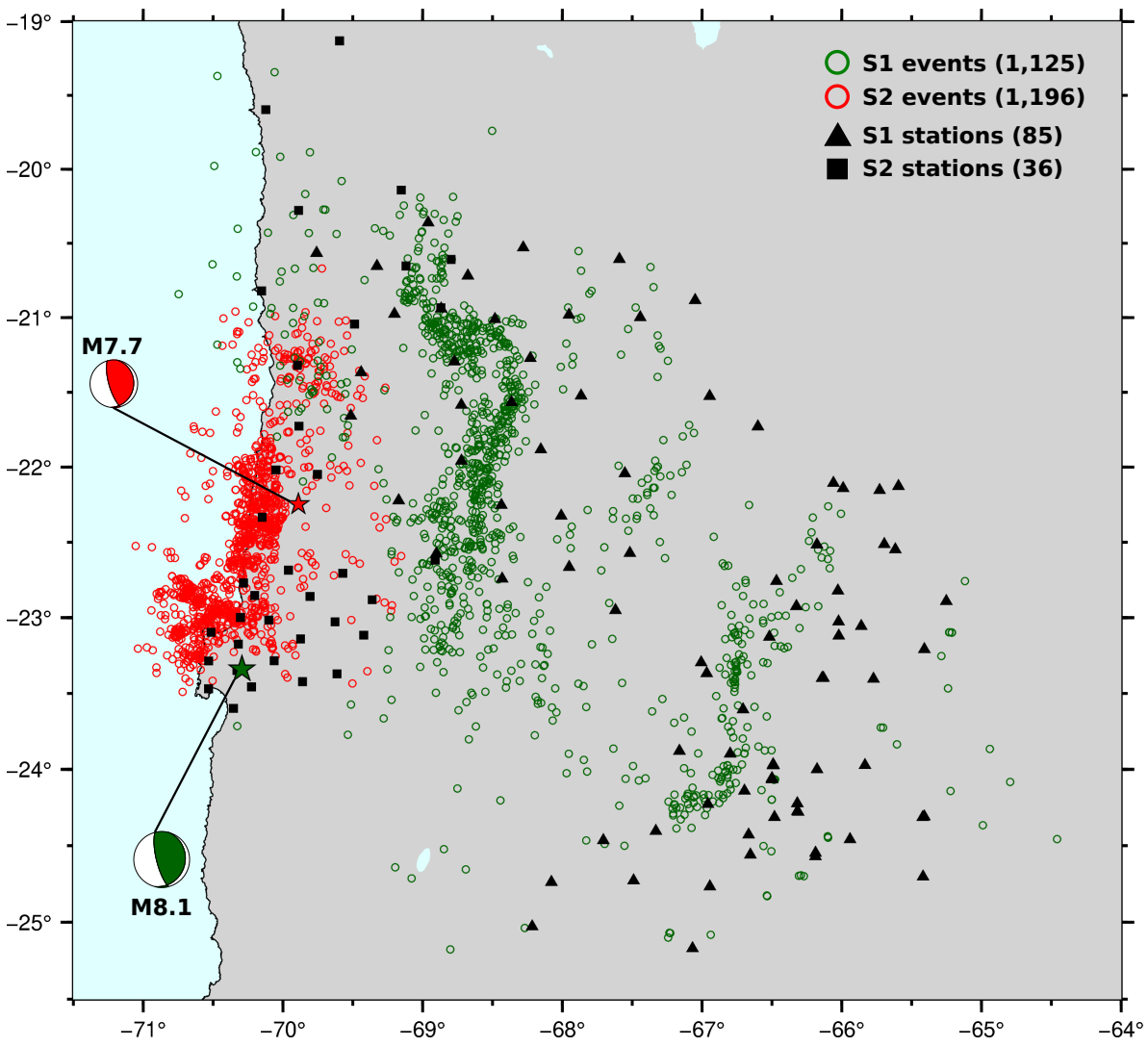

Figure 1. Events datasets from which window samples picked by analysts were extracted for training the phase detection and picking models in DeepPhasePick. Events in S1 and S2 datasets are plotted as green and red circles, respectively. Focal mechanisms (beachballs) and epicenters (stars) of the 1995-07-30 M8.1 Antofagasta and the 2007-11-14 M7.7 Tocopilla earthquakes are plotted in green and red, for reference. Window samples used in this work come from the picked stations plotted as black triangles (from S1) and squares (from S2).

since it may lead to a significant improved performance of the trained model, especially when the available data is limited.

Commonly used hyperparameter optimization approaches, which rely on grid or manual search, have been shown to be less efficient than an optimization based on random search (Bergstra \& Bengio 2012). However, all the above mentioned methods select the subsequently sampled values without an informed criterion. This makes the optimization less effective, since the sampling of hyperparameter values which do not lead to improved performance tends to require a significant amount of time. In contrast, Bayesian optimization selects the next sampled hyperparameters based on previous evaluations. This has proven more efficient in terms of balancing exploration- 
exploitation of the search space, time consumption, and model performance results, compared to random search (Bergstra et al. 2013a; Hinz et al. 2018).

In Bayesian optimization, an objective function is minimized by mapping past evaluations of the hyperparameters to a surrogate probabilistic model of the objective function, which is then more simply optimized instead. In this work, we used a Bayesian approach that optimizes a surrogate model defined by the Tree Parzen Estimator (TPE) algorithm, as implemented in the Python library Hyperopt (Bergstra et al. 2013b). Basically, TPE algorithm applies the Bayes rules to create two different probability distributions for the hyperparameters, depending on the score reached by the objective function. The next set of sampled hyperparameters is then selected based on the expected improvement in the objective function (Bergstra et al. 2011).

We implemented two types of objective functions, which are minimized in order to optimize the training of different adaptive neural network architectures designed to solve two tasks: phase detection and phase picking. These architectures will be described in detail in the next sections. The hyperparameter optimization process was performed using one GPU NVIDIA GeForce RTX 2080. Multiple iterations (trials) were run for each task, with a different hyperparameter configuration being attempted in each trial, until a best-performing trained model, i.e., a model with minimum error, was found. The adaptive neural networks were built in Python using the machine learning framework Keras (Chollet 2015) with Tensorflow as backend (Abadi et al. 2015).

\subsection{Phase detection as a supervised multi-class classification task}

We implemented the phase detection stage as a supervised multi-class classification task, based on an adaptive neural network formed by two blocks of deep layers. The first block is made up of between one and five convolutional layers, which extract abstract representations (features) from three-component seismograms input (samples) and help reduce their dimensionality. The output of the convolutional layers is then flattened before entering the second block, formed by between one and four fully-connected dense layers. All the units in a fully-connected layer are connected to all the units in the preceding layer. This allows them to better learn correlated features throughout the input data, rather than only the locally-connected features learned by convolutional layers. 


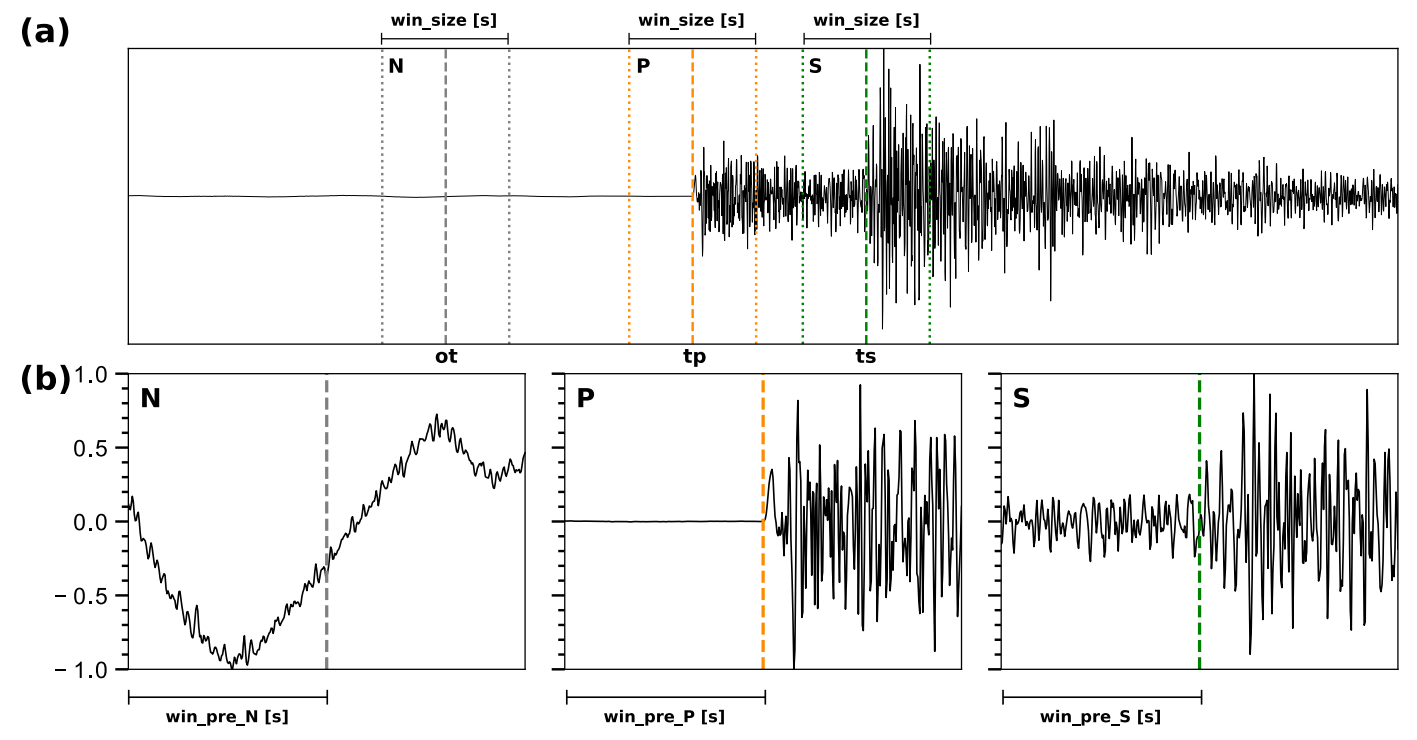

Figure 2. Seismic window extraction. (a) Example of an event waveform, outlining how the seismic window of three classes $(\mathrm{N}, \mathrm{P}, \mathrm{S})$ are extracted for the optimized training of the phase detection model. N-, P-, and S-class windows are extracted, respectively, in the vicinity of the event origin time (ot), and the true P$(t p)$ and S- $(t s)$ onset times found by analysts. The hyperparameter win_size [s] defines the length of the extracted windows, which is equal for the three classes. (b) Zoom into the three-class windows. The starting time of the window before $o t, t p$ and $t s$ is defined by the hyperparameters $f r a c_{-} p r e_{-} N$, frac_pre_ $P$, and frac_pre_S for classes N, P and S, respectively. Here win $_{-} p r e_{-} C=w_{i n \_s i z e} \times f r a c_{-} p r e_{\_} C$, where $C=$ $N, P$ or $S$.

Hence, dense layers are well suited for the final classification stage in the adaptive network. Since the network is adaptive, the number of layers in each block, as well as specific layer variables such as the number of convolutional filters or dense units, are adjusted during the hyperparameter optimization.

We chose to use depthwise separable 1D convolutional layers for the feature extraction in the first block. Depthwise separable convolutional layers implement a 2-step convolution process, first performing independent convolutions on each channel of the input data and then combining individual channel outputs through a pointwise (1x1) convolution (Chollet 2017). This type of convolution is appropriate for learning patterns from multi-component seismic data, such as 1D amplitude time series from seismic waveforms, since different features can be extracted from the three input channels (the three components of each seismogram) independently. This may help the network extract specific patterns from, e.g., the two horizontal components, in order to better 
identify S phases. Furthermore, compared to standard convolutional layers, depthwise separable layers have the additional advantage of helping reduce model overfitting, since they have fewer weights to adjust, and therefore require fewer calculations. This further reduces the overall computational cost, since less calculation time is required to complete the multiple iterations over different network architectures involved in the hyperparameter optimization.

Outputs from each of the convolutional and dense layers in the network, except from the output of the final dense layer, are passed through a layer that applies a non-linear activation function that can be either a rectified linear unit (ReLU; Nair \& Hinton 2010) or a sigmoid, according to the hyperparameter selection. Additional Batch Normalization (Ioffe \& Szegedy 2015) and Dropout (Srivastava et al. 2014) layers are stacked to the activation outputs in both blocks. In the convolutional block, a further 1D Max Pooling layer is applied between each Batch Normalization and Dropout layer. The output of the final dense layer is passed instead through a softmax activation function. This outputs a vector of three probabilities, adding up to 1.0, each expressing the likelihood of a sample belonging to one of the three possible classes tested: P, S, or N. The greatest among these probabilities determines the predicted class of the three-component record.

Prior to initializing the optimized training, we randomly extracted an independent test set consisting of 1,440 P, S and $\mathrm{N}$ three-component time series windows, made up of 888 records from S1 and 552 records from $\mathrm{S} 2$ datasets for each class, so as to keep the proportion of samples present in each dataset. In order to reduce the possibility of biased phase classification due to an imbalanced class distribution in our dataset $(39 \% \mathrm{P}, 39 \% \mathrm{~N}, 22 \% \mathrm{~S})$, the remaining data was balanced out at each optimization trial by randomly discarding the surplus samples of the over-represented P and $\mathrm{N}$ classes. Then, the balanced data was shuffled and assigned to the training and validation sets before carrying out the model training. The resulting training, validation, and test sets added up to $75 \%(32,393), 15 \%(6,478)$, and $10 \%(4,320)$ of the total balanced data samples respectively. During the training, features of the waveforms were learned from the training set and the weights in the network layers were adjusted based on this. The updated model was then used to predict the sample classes in the validation set, and adjust the model hyperparameters accordingly. The final performance of the best trained model was evaluated using the test set. We assigned to each sample 
in the training, validation and test sets, binary vectors representing the sample class as follows: [1, $0,0]$ for $\mathrm{P}$ class $[0,1,0]$ for $\mathrm{S}$ class, and $[0,0,1]$ for $\mathrm{N}$ class. These vectors were used as the known labels which were compared with the vector of class probabilities predicted by the model.

Before entering the network, the seismic records in the training, validation and test sets were linearly detrended, resampled at $100 \mathrm{~Hz}$, and then normalized by the maximum amplitude across the three waveform components. We included additional preprocessing criteria as optimizable hyperparameters. First, the hyperparameter pre_mode controls the type of filter applied to the seismic data, which can be band-pass $(2-10 \mathrm{~Hz})$, high-pass $(>0.2 \mathrm{~Hz})$ or no filter. The length of the extracted seismic windows is given by the hyperparameter win_size (2-5 [s]), which we imposed to be equal for the three classes. This makes data manageable by the network during the training. Finally, three additional hyperparameters varying between 0.2 and 1.0 ( frac_pre_N, frac_pre_P, and frac $_{-}$pre $e_{-} S$ ), define the fraction of the extracted windows placed before the event origin time, the true P-phase onset and the true S-phase onset for classes N, P, and S, respectively (see Fig. 2).

The total space of hyperparameters searched during the optimization is presented in Table 1 . In order to search for the best-performing network trained in detecting seismic phases, we ran 1,000 hyperparameter optimization trials ( $\sim 9$ minutes per trial). In each trial we used the validation accuracy as the optimizable metric and we trained the model for up to 60 epochs using the categorical cross-entropy loss function. To speed up the training process, we additionally applied an early stopping callback that stopped the training if the validation accuracy did not increase in 6 epochs.

\subsection{Phase picking as a supervised sequence binary classification task}

As mentioned previously, phase picking in DeepPhasePick uses two optimized adaptive network architectures, each formed by one or two BLSTM layers, which we implemented as a supervised sequence binary classification task.

We trained a first model for picking $\mathrm{P}$ phases, using as input the amplitude time series taken from vertical-component seismograms. A second model was trained for picking S phases, based on the amplitude time series of the two horizontal-component records. We trained each model to 
Table 1. Hyperparameter search space optimized during the training of phase detection models. The second column lists the hyperparameter values that could be sampled during the optimization. The third column shows the best-performing value found after 1,000 trials. The hyperparameter pre_mode defines the type of filter applied to the seismic data before entering the network as follows: pre_mode $=1,2$, 3 correspond to no filter applied, band-pass filter (2-10 Hz), and high-pass filter $(>0.2 \mathrm{~Hz})$, respectively. Hyperparameters win_size, frac_pre_P, frac_pre_S, and frac_pre_N control the length and time position of the extracted window samples used for the training, as described in the main text and Fig. 2. One additional hyperparameter, not shown here, allows the number of filters in a subsequent convolutional layer to be equal than or twice the value in the current layer.

\begin{tabular}{|c|c|c|}
\hline Hyperparameter & Range tested & Best-performing value \\
\hline \multicolumn{3}{|c|}{ Block of convolutional layers } \\
\hline Number of layers & {$[1,2,3,4,5]$} & 5 \\
\hline Number of filters & {$[2,4, \ldots, 30,32]$} & $12,24,48,96,192$ \\
\hline Kernel size & {$[3,5,7,9,11,13,15,17,19,21]$} & $17,11,5,9,17$ \\
\hline Activation function & [ReLU, sigmoid] & ReLU, ReLU, ReLU, ReLU, sigmoid \\
\hline Dropout & {$[0.2,0.25,0.30,0.35,0.4,0.45,0.5]$} & $0.25,0.25,0.3,0.4,0.25$ \\
\hline \multicolumn{3}{|c|}{ Block of dense layers } \\
\hline Number of layers & {$[1,2,3,4]$} & 1 \\
\hline Number of units & {$[50,100,150,200,250,300]$} & 50 \\
\hline Activation function & {$[$ ReLU, sigmoid] } & ReLU \\
\hline Dropout & {$[0.2,0.25,0.30,0.35,0.4,0.45,0.5]$} & 0.2 \\
\hline \multicolumn{3}{|c|}{ Model training } \\
\hline Optimizer & [Adam, SGD, RMSprop] & RMSprop \\
\hline Learning rate & {$[1 \mathrm{e}-05,1 \mathrm{e}-04,1 \mathrm{e}-03,1 \mathrm{e}-02,1 \mathrm{e}-01]$} & $1 e-03$ \\
\hline Batch size & {$[50,60, \ldots, 190,200]$} & 50 \\
\hline \multicolumn{3}{|c|}{ Data preprocessing and seismic window extraction } \\
\hline pre_mode & {$[1,2,3]$} & 1 \\
\hline win_size $[s]$ & {$[2.0,2.2, \ldots, 4.8,5.0]$} & 4.8 \\
\hline frac_pre_P & {$[0.2,0.3,0.4,0.5,0.6,0.7,0.8,0.9,1.0]$} & 0.7 \\
\hline frac_pre_S & {$[0.2,0.3,0.4,0.5,0.6,0.7,0.8,0.9,1.0]$} & 0.5 \\
\hline frac_pre_N & {$[0.2,0.3,0.4,0.5,0.6,0.7,0.8,0.9,1.0]$} & 0.9 \\
\hline
\end{tabular}


learn patterns from seismic data that account for the transition from noise to signal in both $\mathrm{P}$ and $\mathrm{S}$ phases from local earthquakes, therefore allowing identification of P and S time onsets. BLSTMbased networks are suitable for such task, since they are able to extract and retain meaningful features while processing input seismic sequences in both chronological and anti-chronological order, hence learning dependencies between phase onsets and neighboring seismic patterns. A final dense layer in both picking models applies a sigmoid activation function, which outputs the probability of the timesteps in the time series corresponding to either noise or seismic signal.

Before carrying out the model training, $10 \%$ of the window samples were randomly extracted as an independent test set. As in the phase detection stage, the test set includes a proportional amount of records from S1 and S2 datasets. The remaining samples were first shuffled and then subdivided into training (65\%) and validation (25\%) sets. Altogether, this corresponds to 16,670 training, 6,412 validation, and 2,565 (1,625 from S1, and 940 from S2) test one-component samples for P-picking; and 9,358 training, 3,599 validation, and 1,440 (808 from S1 and 632 from S2) test two-component samples for S-picking. As in the phase detection stage, weights and hyperparameters of the models were adjusted based on the training set and validation set, respectively. The test set was then used to evaluate the performance of the best trained models. The window samples were defined based on the optimized hyperparameter values for the phase detection model, namely the window length (win_size) and its relative position with respect to the true onset (frac_pre_P and frac $_{-} \mathrm{pre}_{-} S$ ). This allows the integration of phase detection and phase picking in a common two-stage workflow, with the aim of making the overall process more efficient, since both stages share already optimized information.

However, instead of using the above-described seismic windows, we trained and validated the picking models on versions of them, which were randomly shifted in time by a range between -5.0 and $5.0[\mathrm{~s}]$ around the true $\mathrm{P}$ and $\mathrm{S}$ onsets. This allows the networks to better learn data patterns in the vicinity of the phase onsets, and so preventing the model from memorizing only fixed onset times. A similar approach has been applied by Ross et al. (2018a) by using an artificially augmented number of samples to train a CNN network as regressor for P-phase picking. In the test 
set, we kept both the original time windows and their shifted versions, in order to test the detection capacity of the trained models in both scenarios.

So as to perform the model training, we assigned to each input sample a binary vector of length equal to the sample window length. This vector is a binary representation of the ground truth, where all the 0s correspond to timesteps associated to noise, and all the 1s representing signal, so that the first 1 represents the timestep of the true phase onset. Once trained, the models output a vector of the same length, which contains numbers between 0 and 1.0 corresponding to binary class probabilities: $<0.5$ for timesteps predicted as noise and $>0.5$ for timesteps predicted as signal. Thus, we can determine the predicted phase time onset in a given sample as the timestep where the output probabilities first rise above 0.5 , indicating a change from noise to signal.

We ran 50 hyperparameter optimization trials for the $\mathrm{P}$ - ( $\sim 130$ minutes per trial $)$ and S-phase ( $\sim 50$ minutes per trial) picking tasks. Table 2 presents the hyperparameter space searched during the optimization. We optimized on the validation accuracy metric, using the binary cross-entropy loss function and the Adam stochastic optimization algorithm (Kingma \& Ba|2014) in the training. The training was carried out using the same number of epochs and similar early stopping as in the phase detection stage.

\subsection{From predicted phase probabilities to preliminary onsets}

By applying our best-performing models trained for both phase detection and phase picking on continuous seismic data, we were able to obtain the time onsets of $\mathrm{P}$ and $\mathrm{S}$ phases originating from local earthquakes. In this section we explain how we first obtained preliminary time onsets in the phase detection stage. The next section describes how we refined them in the phase picking stage to estimate the final phase onsets and their associated uncertainties.

First, we used the optimized phase detection model to predict the class probabilities of threecomponent moving windows. By iteratively shifting these windows along the continuous seismic waveforms, we obtained a discrete probability time series of each class. The length of the moving window is given by the optimized hyperparameter win_size. We assigned the predicted probability at the position within the window defined by the optimized hyperparameters frac_pre_N, 

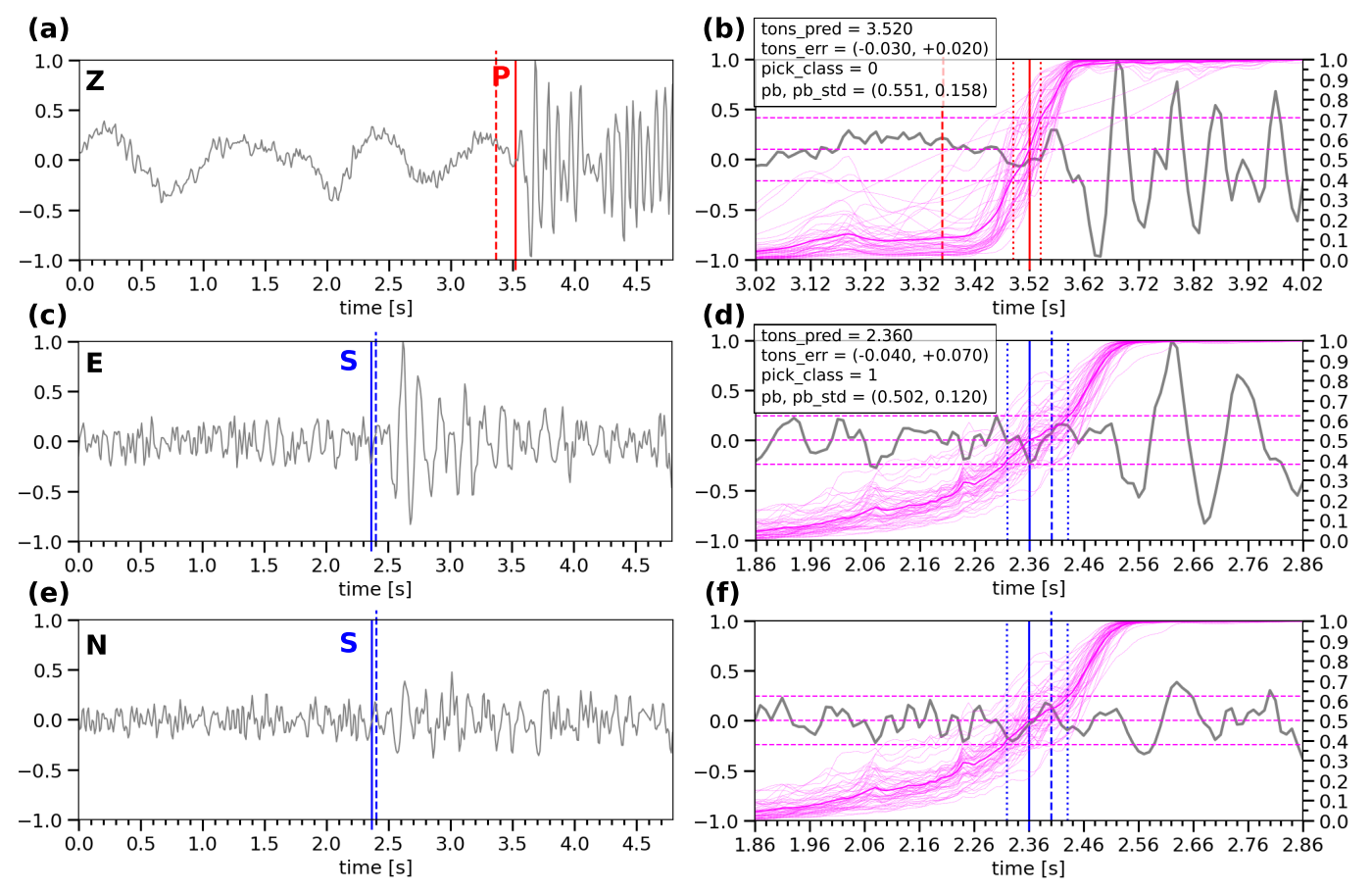

Figure 3. Estimation of time onset and associated uncertainty for predicted $P$ (a-b) and $S$ (c-f) phases. (a), (c), (e) Examples of predicted Z, E, N (Vertical, East, North) window samples. Red and blue dashed lines represent the preliminary $\mathrm{P}$ and $\mathrm{S}$ onsets predicted in the phase detection stage, which were refined in the phase picking stage (red and blue solid lines). (b), (d), (f) Zoom in centered on the refined P and S time onsets (tons_pred $[\mathrm{s}])$, estimated by applying MCD $(\mathrm{T}=50)$ inference. Predicted class probabilities for each MCD prediction are plotted as magenta curves (right y-axis); bold curve represents the mean probability. Magenta horizontal dashed lines indicate the mean $(p b)$ and one standard deviation ( $\left.p b \_s t d\right)$ of the class probabilities at the onset time. As explained in the main text, from $p b$ and $p b \_s t d$, we can obtain tons_pred and its uncertainty (tons_err [s]), outlined by red and blue vertical dotted lines before and after the refined P- and S-phase onsets. Based on the weighting class scheme defined in Table 3 , P and S phases shown here correspond to picks of class 0 and 1 , respectively.

frac_pre_P, and $f r a c_{-} p r e_{-} S$ for the respective classes (see Table 1 and Fig. 2). The timestep between consecutive moving windows is a user-defined parameter that controls the resolution of the probability time series.

Next, we declared a P-phase search window between the time where the P-class probability time series $\left(p b_{-} P\right)$ rises above certain trigger threshold $p b_{-} P_{-} t h 1$ and the time where $p b_{-} P$ decreases below a certain detrigger threshold $p b_{-} P_{-} t h 2$. We estimated a preliminary P-phase onset as the time of the maximum $p b_{-} P$ within the search window. For waveforms where a preliminary $\mathrm{P}$ onset is found, we followed a similar approach to define a preliminary S-phase onset at the time 
Table 2. Hyperparameter search space optimized during the training of P- and S-phase picking models. The second column lists the hyperparameter values that could be sampled during the optimization. The third and fourth columns show the best-performing values found after running $50 \mathrm{P}$ - and S-phase optimization trials.

\begin{tabular}{|c|c|c|c|}
\hline Hyperparameter & Range tested & Best value (P-picking) & Best value (S-picking) \\
\hline \multicolumn{4}{|c|}{ Recurrent BLSTM layers } \\
\hline Number of layers & {$[1,2]$} & 2 & 2 \\
\hline Units & {$[50,60, \ldots, 190,200]$} & 100,160 & 20,30 \\
\hline Dropout & {$[0.2,0.25,0.30,0.35,0.4,0.45,0.5]$} & $0.2,0.35$ & $0.25,0.45$ \\
\hline Recurrent Dropout & {$[0.2,0.25,0.30,0.35,0.4,0.45,0.5]$} & $0.2,0.25$ & $0.35,0.25$ \\
\hline \multicolumn{4}{|c|}{ Model training } \\
\hline Learning rate & {$[1 \mathrm{e}-05,1 \mathrm{e}-04,1 \mathrm{e}-03,1 \mathrm{e}-02,1 \mathrm{e}-01]$} & $1 \mathrm{e}-041 \mathrm{e}-02$ & 1e-02 \\
\hline Batch size & {$[50,60, \ldots, 190,200]$} & 60 & 50 \\
\hline
\end{tabular}

of the maximum S-class probability time series $\left(p b_{-} S\right)$, within an S-phase search window delimited by the time interval that satisfies the condition $p b_{-} S_{-} t h 1<p b_{-} S<p b_{-} S_{-} t h 2$. Cases where $p b \_P$ and $p b \_S$ overlap or are close enough in time at values above the trigger thresholds cannot be handled solely with the previous criteria. We included few additional conditions, based on the predicted probabilities, to deal with this and other particular scenarios. These conditions can be optionally activated by the user and depend on a few user-defined parameters (see Text S1).

\subsection{Estimation of final phase onset times and their uncertainties}

The preliminary $\mathrm{P}$ and $\mathrm{S}$ onsets were defined based on probabilities returned by a model trained for recognizing seismic phases, rather than for actually picking them, and therefore need to be refined. To this end, we used the search windows corresponding to the preliminary P-and S-phase onsets as "picking windows". On these picking windows, we then applied the optimized models trained for P- and S-picking to estimate the respective phase onsets as well as the associated uncertainties.

Uncertainties were determined by applying the stochastic regularization technique Monte Carlo Dropout (MCD). It has been shown that using dropout for training a deep neural network can be 
interpreted as a Bayesian approximation of a Gaussian process, hence MCD can be used as an approximation of Bayesian variational inference (Gal \& Ghahramani 2016a|b). This means that a model posterior distribution for a given sample can be represented by multiple (T) predictions made by the network with dropout. Thus, the value and uncertainty of the predicted sample can be obtained as the mean and standard deviation of the $\mathrm{T}$ inferences.

In practice, we implemented MCD by performing T model predictions with dropout on. In this case, since phase picking is treated as a sequence binary classification task, MCD generates a collection of predicted class probabilities of the timesteps in an input time series. This collection is an approximation of the model posterior distribution for that input sample, which is described by the mean and standard deviation of the T class probabilities at each of the sample timesteps.

Then, we estimated the predicted phase time onset (tpred) for the sample as the first timestep in which the mean of the class probabilities rises above $0.5(p b)$, which indicates the predicted transition from noise to signal. The standard deviation of the class probabilities at tpred ( $p b_{-} s t d$ ) represents the variability of the predicted probability at the assumed time onset. We propose that a reasonable first order estimation for the time onset uncertainty can be determined as the interval of timesteps delimited before and after tpred by the projection of $p b \_s t d$ on the mean class probability (see Fig. 3). The resulting time uncertainty is asymmetric and inversely proportional to the slope of the mean predicted probability curve around tpred. That is, the steeper the decrease/increase in the predicted probability before/after tpred, the lower the time uncertainty, as one might intuitively expect from an abrupt, easy-to-pick change from noise to signal. Based on these estimated pick uncertainties, we defined the weighting class scheme for the phases $\mathrm{P}$ and $\mathrm{S}$ shown in Table 3 , which was adapted from (Sippl et al.|2013).

\section{RESULTS}

\subsection{Phase detection model optimization}

Fig. 4 summarizes how the six main hyperparameters in the phase detection model evolved over the 1,000 optimization trials attempted. For the majority of the main hyperparameters, the most frequently sampled hyperparameter value (higher histogram bins) did not coincide with the 

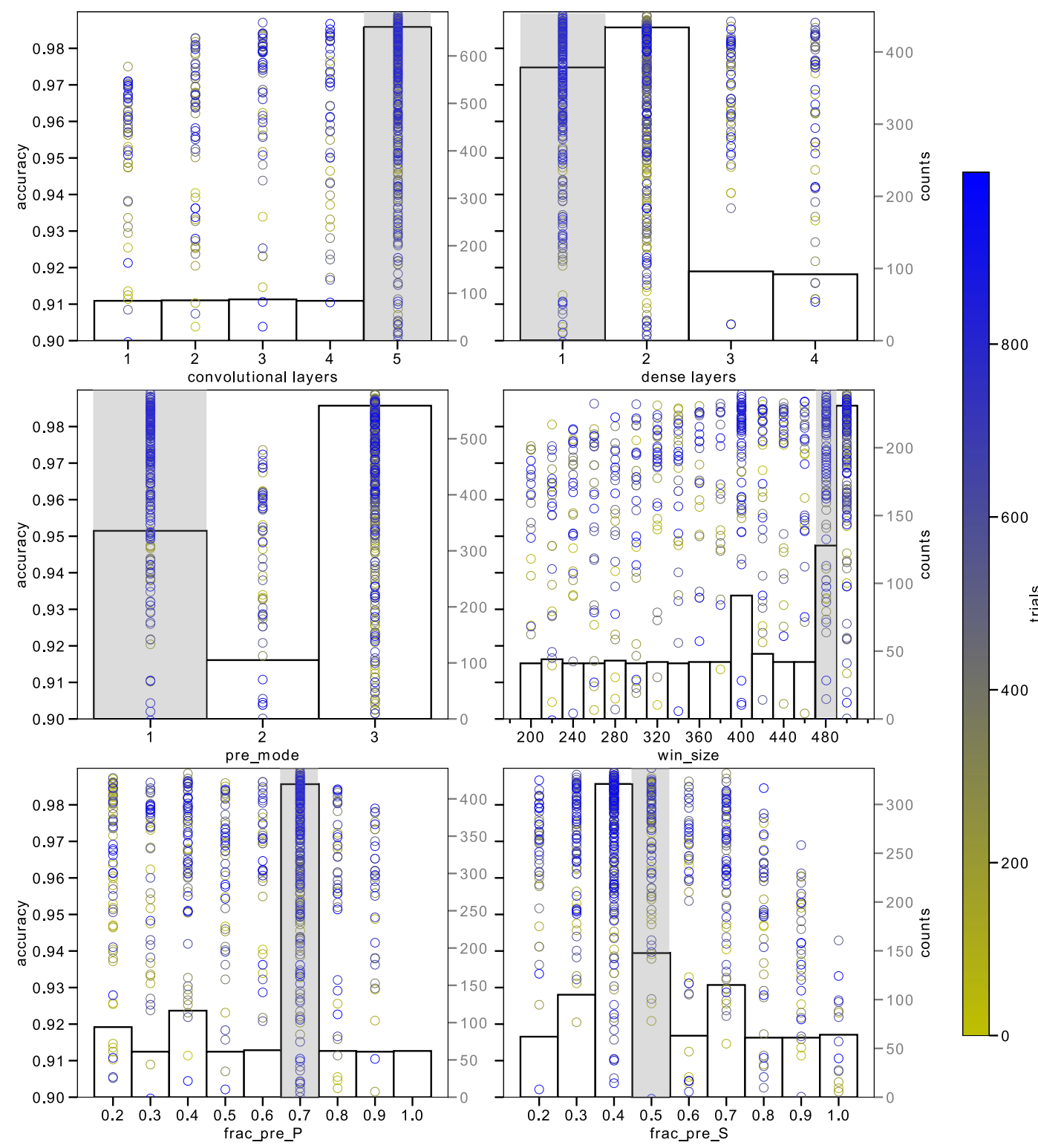

Figure 4. Distribution over the 1,000 optimization trials of the six main hyperparameters used in the phase detection model training. Subplots show sampled hyperparameter values colored by trial number and plotted versus the model accuracy reached at that trial (left y-axis). The distribution of the hyperparameter values sampled over the trials is presented as a histogram (right y-axis). A gray shaded bin in the histogram represents the best-performing hyperparameter value, that is, the value with which the model reached the highest accuracy.

value that led to the highest model accuracy found after 1,000 trials (shaded histogram bin), although this correlation was observed for the number of convolutional layers and $f r a c_{-} p r e \_P$.

Remarkably, as can be seen from the subplots in Fig. 5, the best hyperparameter values or the most frequently sampled values (when both did not coincide) formed clusters at several times 


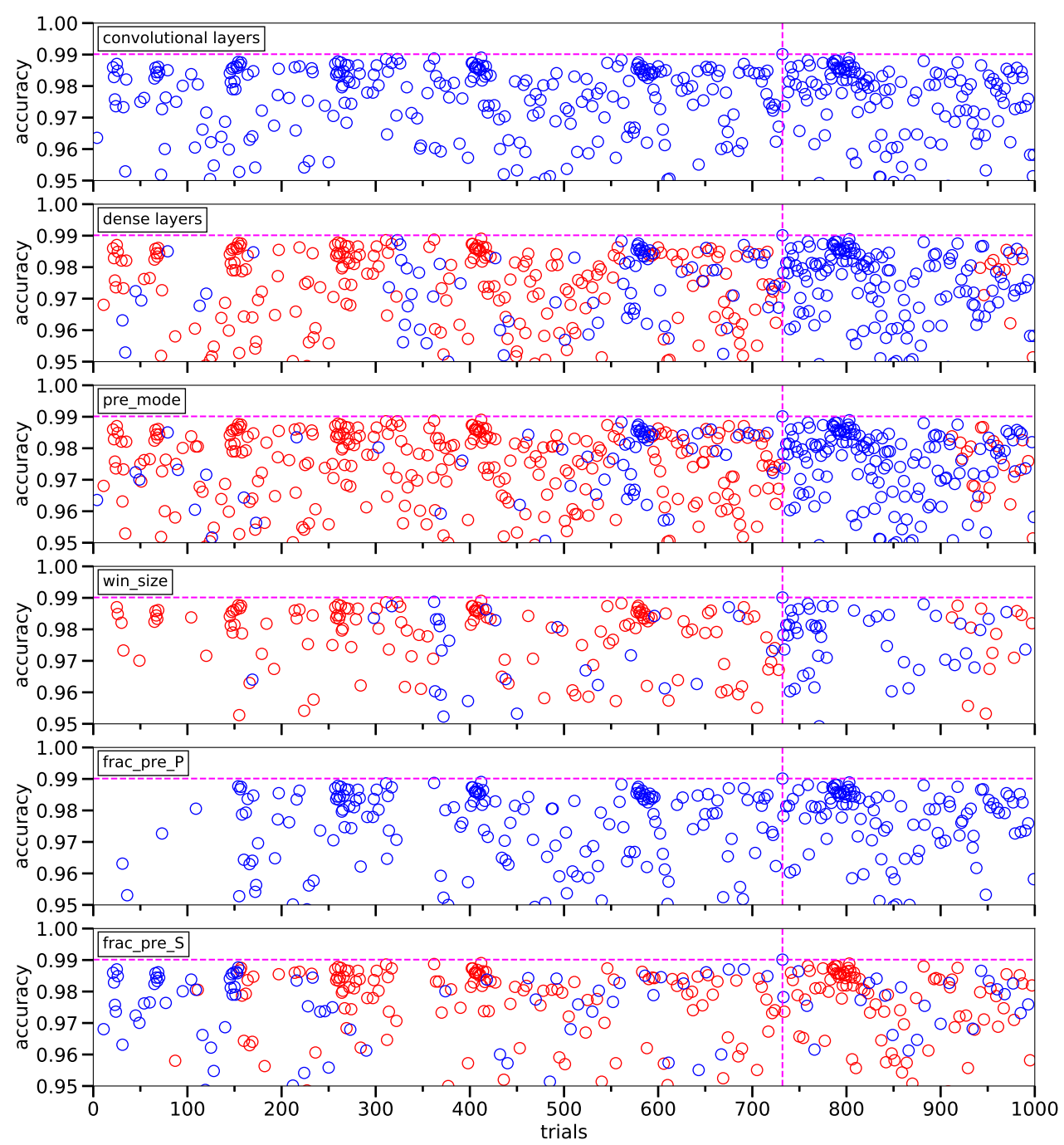

Figure 5. Each subplot depict in detail the distribution of the best-performing hyperparameter values (gray shaded bins in histograms of Fig. 4 , blue circles) that led to higher model accuracies $(>0.95)$ over the trials. Values plotted are the accuracies on the validation set, reached at the end of each trial training. For the hyperparameters in which the best-performing value does not coincide with the most frequently sampled value, the latter are plotted as well (red circles). For reference, magenta horizontal and vertical dashed lines mark the highest model accuracy (0.990) reached during the optimization and the trial (732) at which it was achieved, respectively.

(around trials 150, 260, 400, 590, and 800) during the optimization. This suggests that the same set of hyperparameter values was systematically found to be effective in producing highly accurate models during the optimization process, while the rest of the hyperparameters in the network were probably still being adjusted.

The best-performing hyperparameter values indicate that models in which the convolutional 

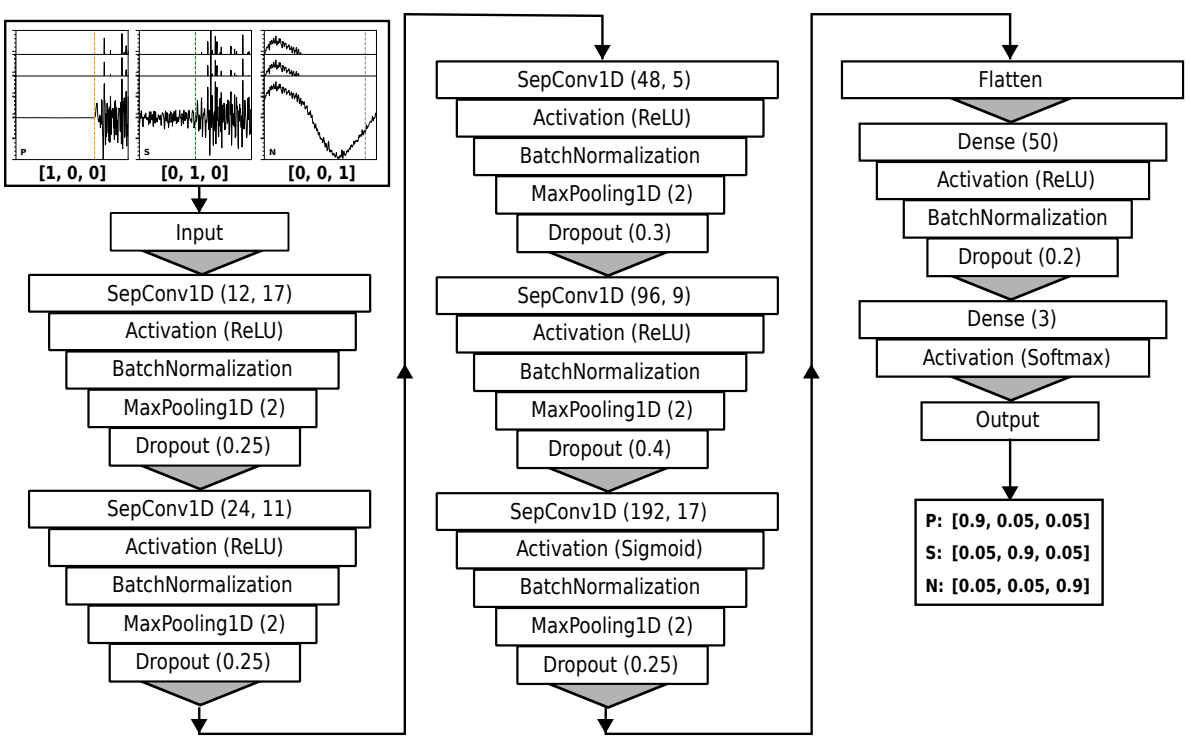

Figure 6. Optimized model architecture trained for phase detection task. The network receives as input class vectors representing the respective accompanying three-component seismogram samples and outputs three-probability vectors indicating the predicted classes $(\mathrm{P}, \mathrm{S}$, or $\mathrm{N})$. The optimized hyperparameters in each block of convolutional and dense layers are shown here and also presented in Table 1.

block was considerably deeper (five convolutional layers) than the dense block (only one or two dense layers) proved to be more accurate. Furthermore, models that learned from band-pass filtered data reached lower training accuracy in comparison to when high-pass filter or no filter was applied, with the latter approach performing the best. Thus, using the whole frequency content proved to be more effective for model training than removing very long period background seismic noise or substantially limiting the frequency content. We note here that quite a few of the data

Table 3. Weighting scheme for $\mathrm{P}$ and $\mathrm{S}$ predicted pick classes. Where terr $=\frac{1}{2}\left(\mid\right.$ tons_err $^{-}|+|$tons_err $\left.^{+} \mid\right)$ is the mean of the absolute time uncertainties calculated before and after the phase onset shown in Figs 3 (b) and (d) insets.

\begin{tabular}{cc}
\hline Quality Class & Time onset error (terr) \\
\hline P0, S0 & terr $<0.05$ \\
P1, S1 & $0.05<$ terr $<0.1$ \\
P2, S2 & $0.1<$ terr $<0.2$ \\
P3, S3 & terr $>0.2$ \\
\hline
\end{tabular}


are from short-period seismometers, hence inherently high-pass filtered $(>1 \mathrm{~Hz})$, which could account for the most frequently sampled value of pre_mode.

Another important constraint in the optimization process seems to be the amount of data available for the network to learn, since longer window samples (480-500 s) clearly led to more correctly predicted phases. $\mathrm{P}$ phases were more accurately predicted when the sample windows include a relatively larger portion of noise (70\%) compared to signal, whereas S phases were better predicted from windows containing similar amount of noise relative to signal (50\%).

Table 1 lists the best-performing values found for each of the optimized hyperparameters, which were used to train the best phase detection model architecture outlined in Fig. 6

\subsection{Predicted phase classes compared to analyst labels}

Figs 7 (a) and (b) show that the accuracy of the best phase detection model smoothly increased on the training set and reached 0.986 after 35 epochs, when the early stopping condition stopped the training. The training loss progressively decreased and reached 0.044 at the end of the training. The accuracy and loss calculated on the validation set followed a similar behavior to the training curves, although showing overall higher accuracy and lower loss due to the use of dropout regularization during training. Dropout disables a certain number of layer units, therefore some information about the input samples is lost and the network attempts to learn based on incomplete data representations. However, during validation all the units are available, thus the network uses its full computational power.

The confusion matrix in Fig. 7(c) shows the overall performance of the best trained model in classifying the classes of the independent test set of 4,320 samples. The cells in this matrix are filled with the predicted classes, which correspond to the highest of the three-class probabilities returned by the model for each sample. An overall very high ratio (0.99) of correct predictions is observed for the three classes, as can be seen from the numbers in the matrix diagonal. Fig. S1 show waveform examples of these correctly predicted P-, S- and N-class samples.

Of the few misclassified samples, only three correspond to $\mathrm{S}$ phases predicted as $\mathrm{P}$, whereas no P phases were mispredicted as $\mathrm{S}$, indicating that the network has a high capability to discriminate 

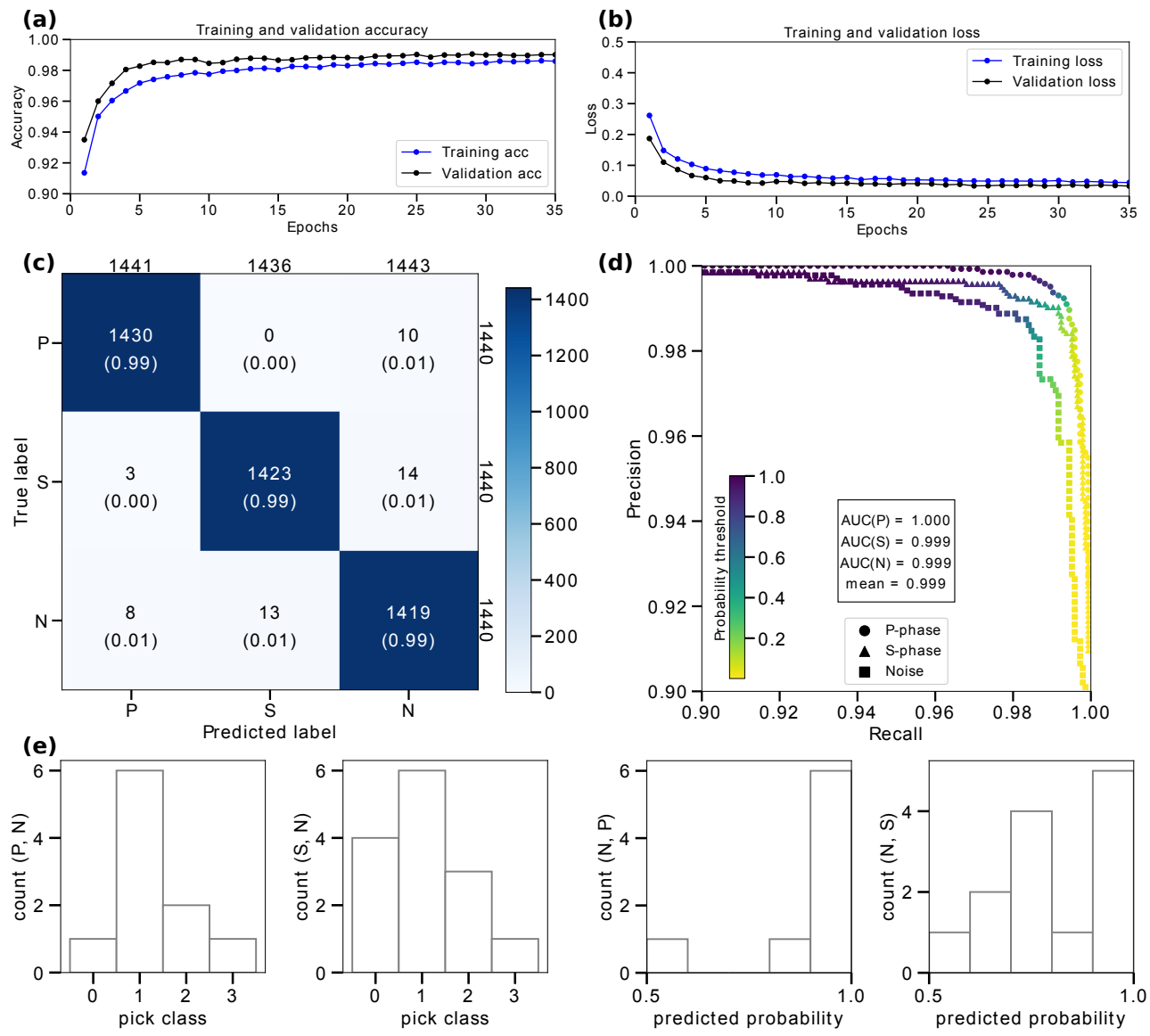

Figure 7. (a-b) Evolution of the training and validation accuracy (a) and loss function (b) over the training epochs of the optimized model trained for phase detection. (c-d) Results from evaluation of the best phase detection model on the independent test set of 4,320 samples, represented in a confusion matrix (c) and a precision-recall curve (d). In (d), AUC represents the area under the precision-recall curve for each class. (e) disaggregated histograms of incorrectly predicted $\mathrm{P}$ or $\mathrm{S}$ phases as noise and presumably misclassified noise samples as $\mathrm{P}$ or $\mathrm{S}$ phases. (P, N) means P-phase samples predicted as Noise, and similarly for other cells in the confusion matrix.

between both classes. Fig. S2 shows one of these misclassified S phases, where the pick made by the analyst was somewhat earlier than the most impulsive change in amplitude. This could be one possible source of misclassification between the two phases, since it may have led to the network misinterpreting the sample as a $\mathrm{P}$ phase.

The remaining mispredictions correspond to either actual P or S phases predicted as noise (24 samples) or presumed noise predicted as $\mathrm{P}$ or $\mathrm{S}$ phases (21 samples). From the samples in the former group, the majority of the $\mathrm{P}(90 \%)$ and $\mathrm{S}(\sim 71 \%)$ samples were assigned pick weights higher or equal than 1 ( $<75 \%$ confidence) by analysts (Fig. 7e). This is indicative of waveforms 


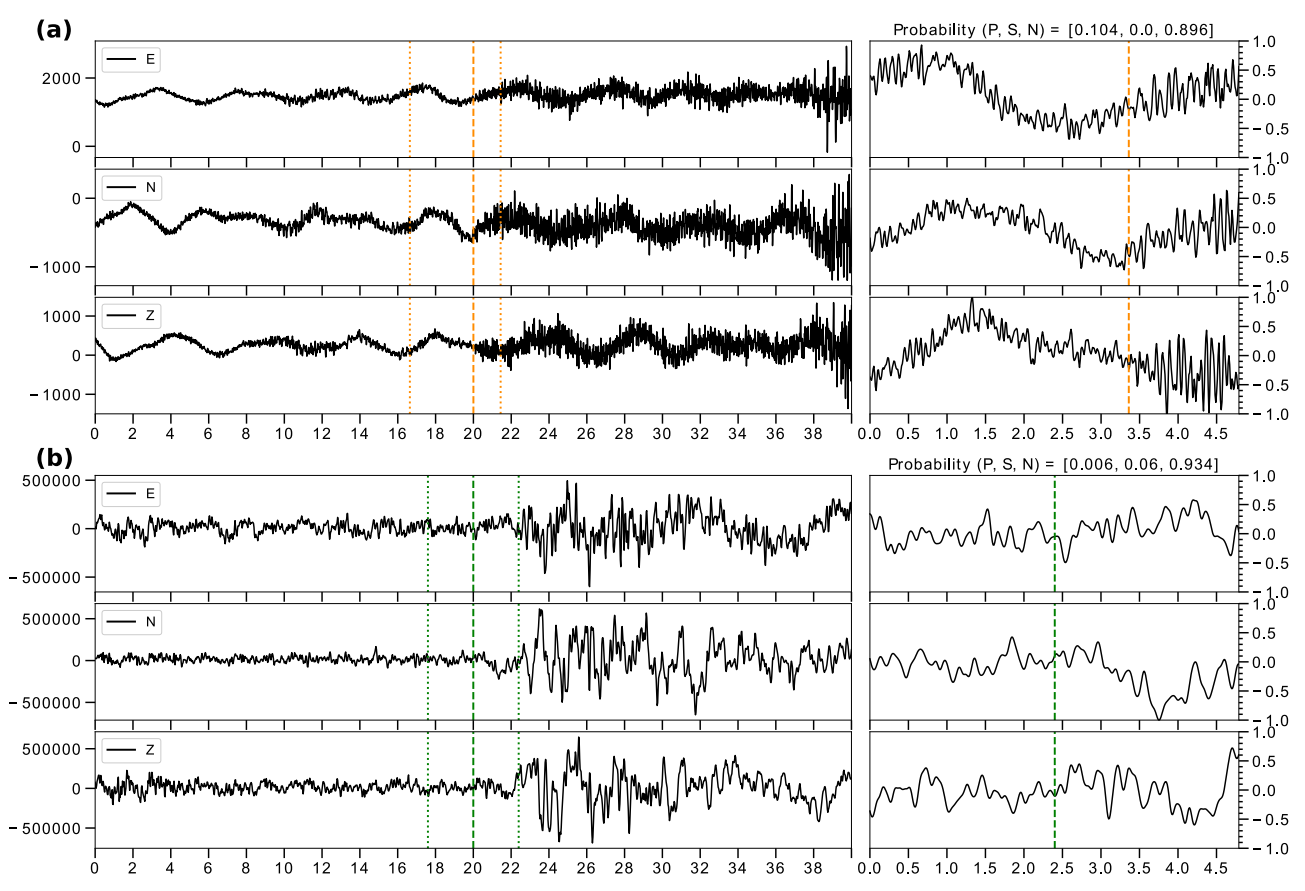

Figure 8. (a) Example of P-phase sample in the test set of 4,320 samples, mispredicted as noise (N). (b) Example of S-phase sample in this test set, mispredicted as noise. The left subplots in (a) and (b) show the three-component seismic wavefrom from which the samples were extracted. The extracted samples, as received by the network, are shown in the right subplots. Examples shown here correspond to P-phase and S-phase samples with analyst's pick weights 2 (50\% confidence) and 1 (75\% confidence), respectively.

with relatively low signal-to-noise ratio (SNR), as can be seen from the examples of mispredicted $\mathrm{P}$ and $\mathrm{S}$ phases shown in Figs 8 (a) and (b), which may cause the model misclassification. In the latter group, a significant majority of the noise samples were predicted as signal with relatively high probability. In fact, of these, $87.5 \%$ of the $\mathrm{P}$ and $\sim 77 \%$ of the $\mathrm{S}$ predictions had a probability higher than 0.7 , with $75 \%$ of the $\mathrm{P}$ and $38 \%$ of the S predictions having a probability higher than 0.9 (Fig. 7e). The examples in Figs 9(a) and (b) demonstrate that these apparently mispredicted samples actually correspond to previously undetected phases, hence evidencing the powerful detection capacity of the model.

The detection capability of the trained model can be further assessed by calculating the precision and recall metrics. Precision, given by TP / (TP + FP), describes how well the model predicts a specific class, by comparing the correct predictions of the class (true positives: TP) with all the predictions made for that class (TP + FP; where FP means false positives). Recall is computed as $\mathrm{TP} /(\mathrm{TP}+\mathrm{FN})$ and reports the ability of the model to identify all the samples of a given class, 

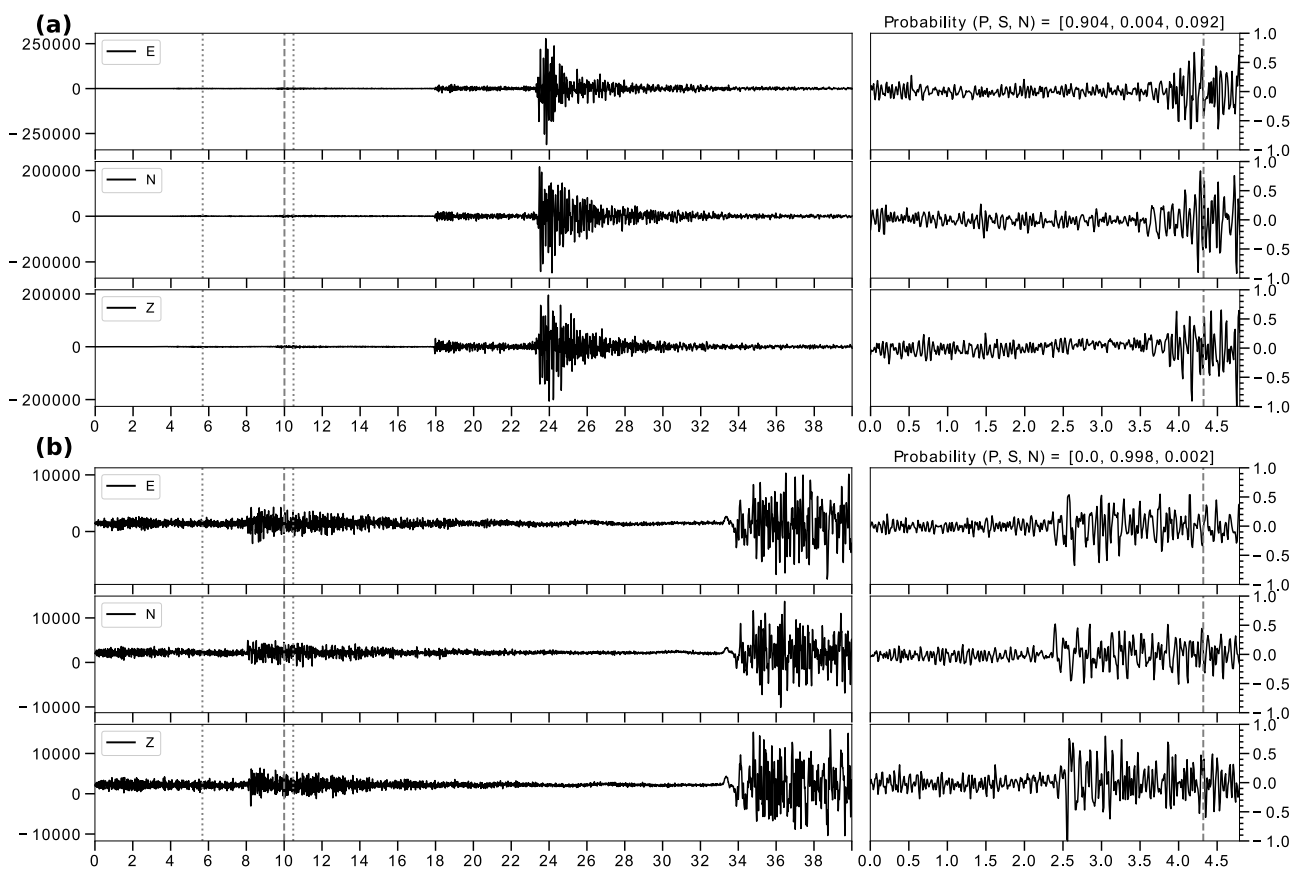

Figure 9. (a) Example of noise (N) sample in the test set of 4,320 samples, seemingly mispredicted as a P-phase. (b) Example of noise sample in this test set, seemingly mispredicted as a S-phase. Subplots are plotted the same way as in Fig. 8. The apparent misclassifications shown here are examples of non-picked phases, which were however detected by the network.

by comparing TP with all the samples of that class in the test set $(\mathrm{TP}+\mathrm{FN}$; where $\mathrm{FN}$ are false negatives).

Precision and recall vary between 0 and 1, where higher values are associated with better performing models. In a precision-recall curve, precision is plotted against recall for different probability thresholds, i.e., the probability above which a sample is predicted to be of a certain class. The more the curve bends toward the point $(1,1)$, and therefore the larger the area under the curve (AUC), the better the model performance. Fig. 7(d) shows the precision-recall curve obtained for the samples in the independent test set. As can be seen here the performance of the model in predicting both P- and S-class samples was very high, with the performance being slightly better for the $\mathrm{P}$ than for the $\mathrm{S}$ class. The model performance on $\mathrm{N}$-class samples was somewhat lower. However, this calculated performance does not take into account any presumed N-class samples where the model detected a real seismic signal. As previously discussed by Ross et al. (2018b), the probability threshold can be tuned so as to improve precision (reducing false positives) or recall (aiming at reducing missed detections), depending on the application. 
(a)

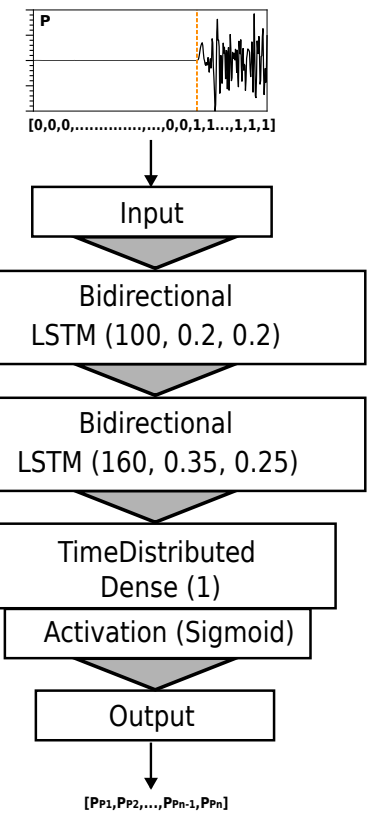

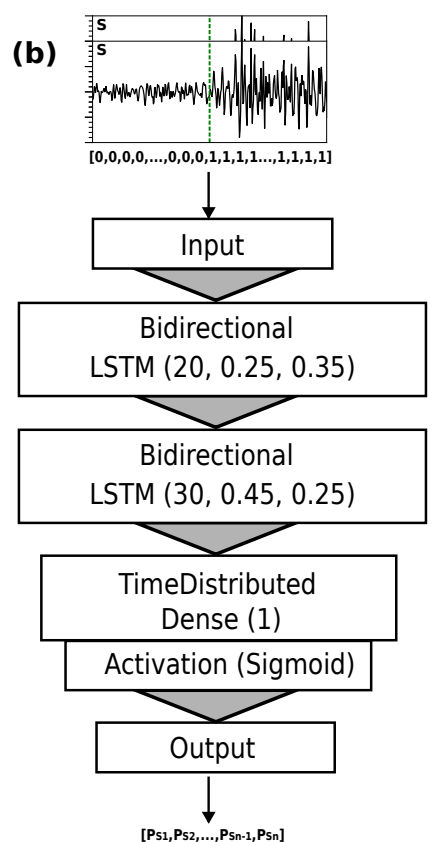

Figure 10. Optimized model architectures trained for P-phase (a) and S-phase (b) picking tasks. The networks receive as input binary vectors representing the noise (0) and signal (1) content in accompanying time series taken from the vertical-component (a) and the two horizontal-component (b) seismograms of the samples used for training. The models output is the probability of the timesteps in input samples corresponding to either noise or seismic signal. The optimized hyperparameters in each BLSTM layer are shown as (Units, Dropout, Recurrent dropout), which are also reported in Table 2 for both models.

\subsection{Predicted phase time onsets compared to analyst picks}

Table 2 shows the best-performing hyperparameter values found during the optimization of the P- and S-phase picking models. These parameters were used to train the best picking model architectures outlined in Figs 10(a) and (b).
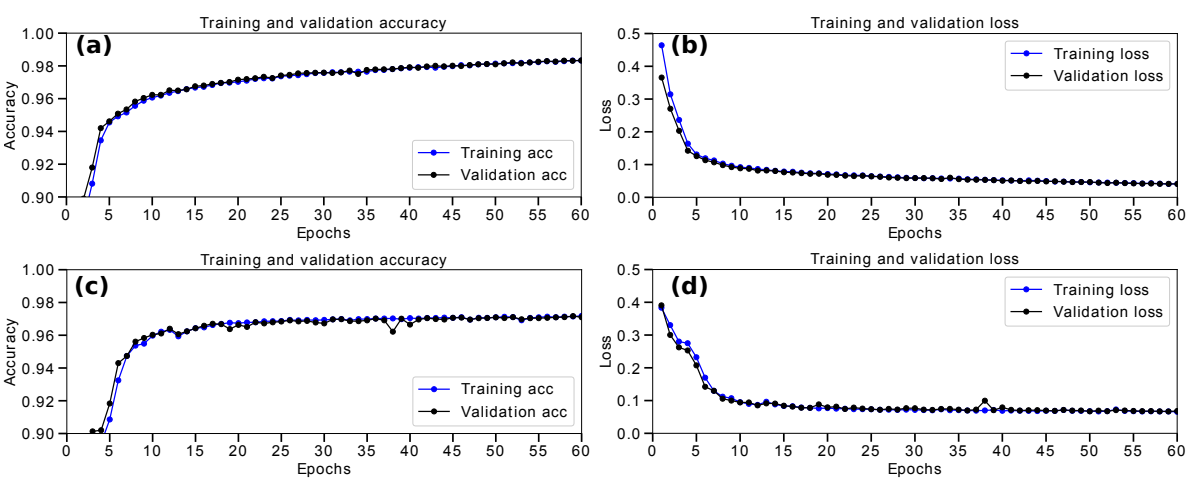

Figure 11. Evolution of the training and validation accuracy and loss function over the training epochs of the optimized model trained for P-phase (a-b) and S-phase (c-d) picking. 

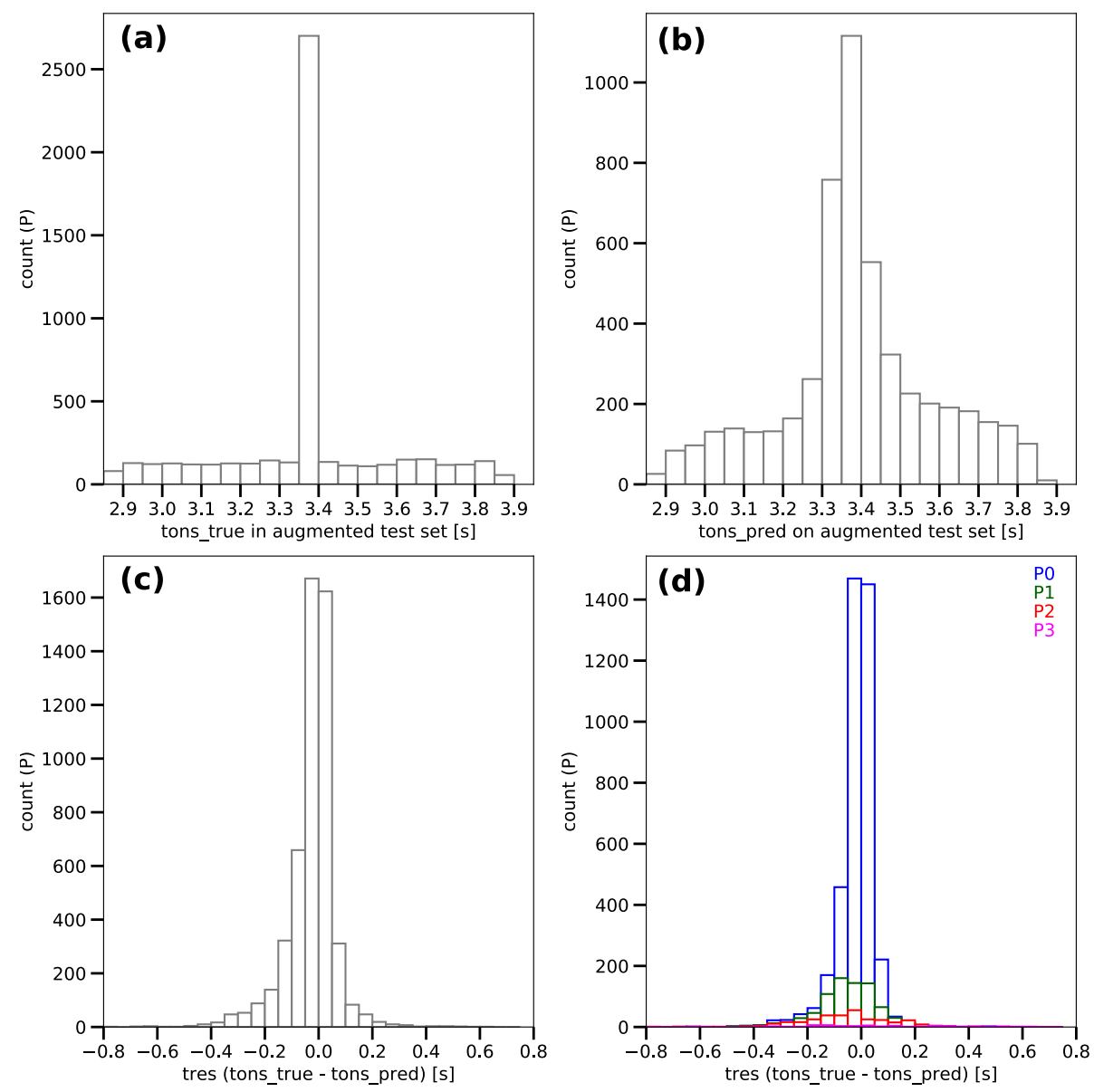

Figure 12. Evaluation of the optimized model trained for P-phase picking on the independent augmented test set of 2,565 x 2 one-component waveform samples. (a) True time onsets (tons_true) from both the actual and shifted samples forming the augmented test set. (b) Predicted time onsets (tons_pred, defined as in Fig. 3) by the best model trained for P-phase picking. (c) Time residuals (tres) distribution, defined as the difference between the true and predicted time onsets. (d) Predicted pick quality classes, as defined by weighting scheme defined in Table 3 .

At the end of the training, the best P-picking model reached higher accuracies $(0.983$ in training) than the accuracies of the S-picking model (0.972 in training). This is also reflected in the training and validation loss functions, which reached a lower value for $P$ phases ( 0.041 in training) than for S phases (0.066 in training) (Fig. 11). As previously explained, the MCD estimation of predicted phase time onsets and their uncertainties requires that the picking models are trained with dropout enabled. For this reason the training and validation curves do not show the difference in accuracy and loss observed for the best phase detection model.

Figs 12 and 13 summarize the performance of the optimized models trained for picking $\mathrm{P}$ 

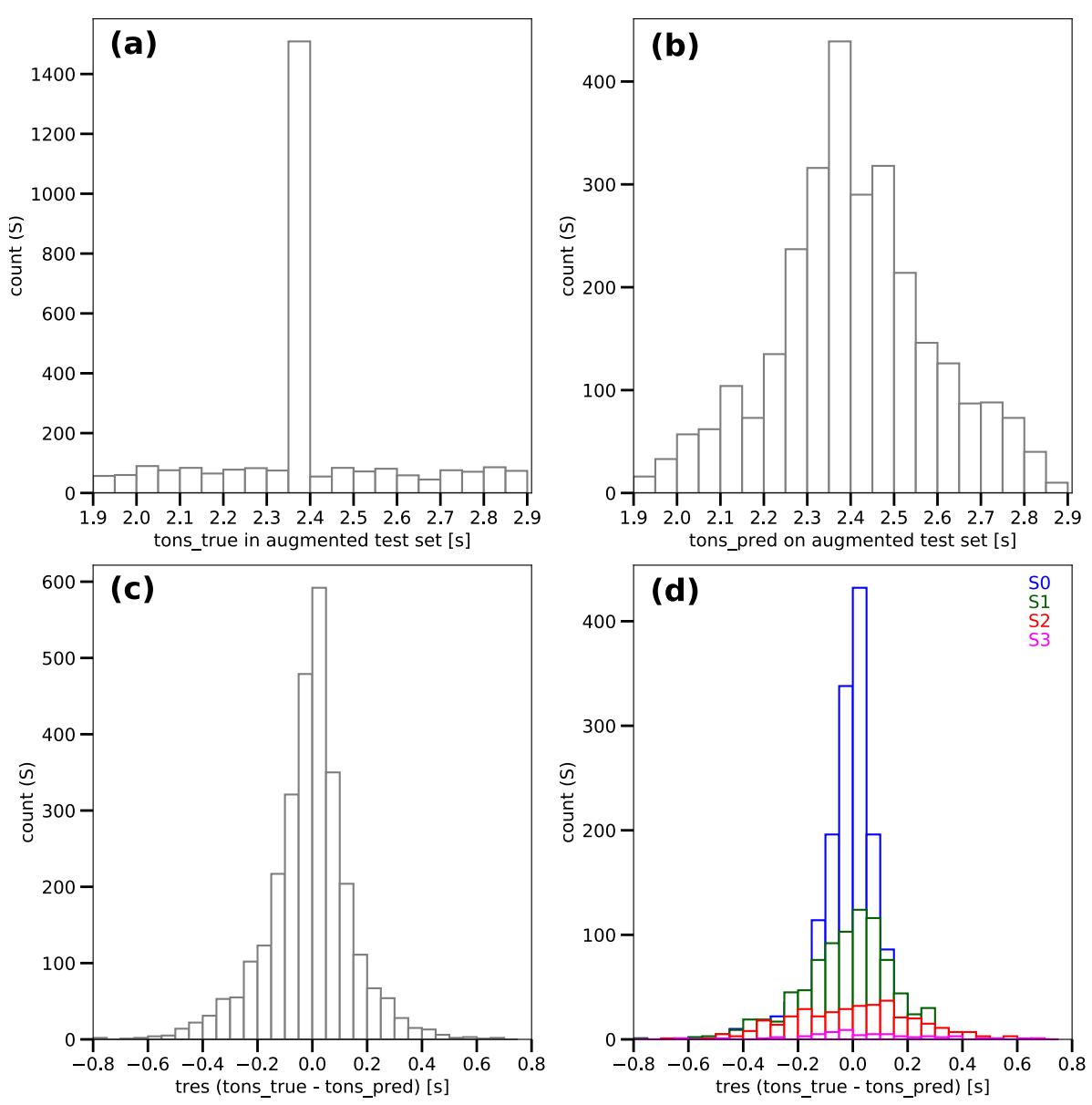

Figure 13. Evaluation of the optimized model trained for S-phase picking on the independent augmented test set of 1,440 x 2 two-component waveform samples. Subplots are similar as in Fig. 12.

and S phases, when evaluated on the independent test sets of 2,565 one-component and 1,440 twocomponent samples respectively. In order to evaluate how the models perform in different possible picking scenarios, we used an augmented test set formed by two groups of samples: 1) the actual waveforms in the original test set, as defined by the optimized hyperparameters of the best phase detection model (win_size, frac_pre_P, and frac_pre_S), and 2) artificial versions of the same waveforms, randomly shifted between -0.5 and $0.5 \mathrm{~s}$ around the true phase onsets made by the analysts.

The slightly higher accuracy reached by the P-phase picking model is reflected in the shape of the true and predicted time onset distributions, which more closely resemble each other for the P-phase (Figs 12a and b) compared to the S-phase (Figs 13a and b). Consequently, a narrower distribution of time residuals was obtained for $\mathrm{P}$ phases compared to $\mathrm{S}$ phases (Figs $12 \mathrm{c}$ and $13 \mathrm{k}$ ). 
(a)
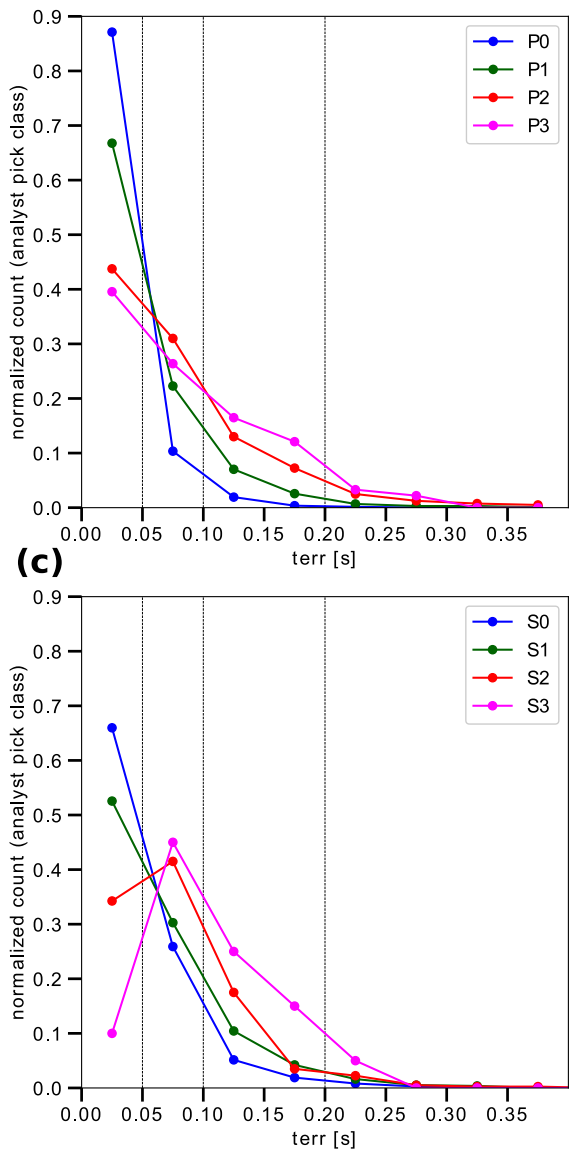

(b)
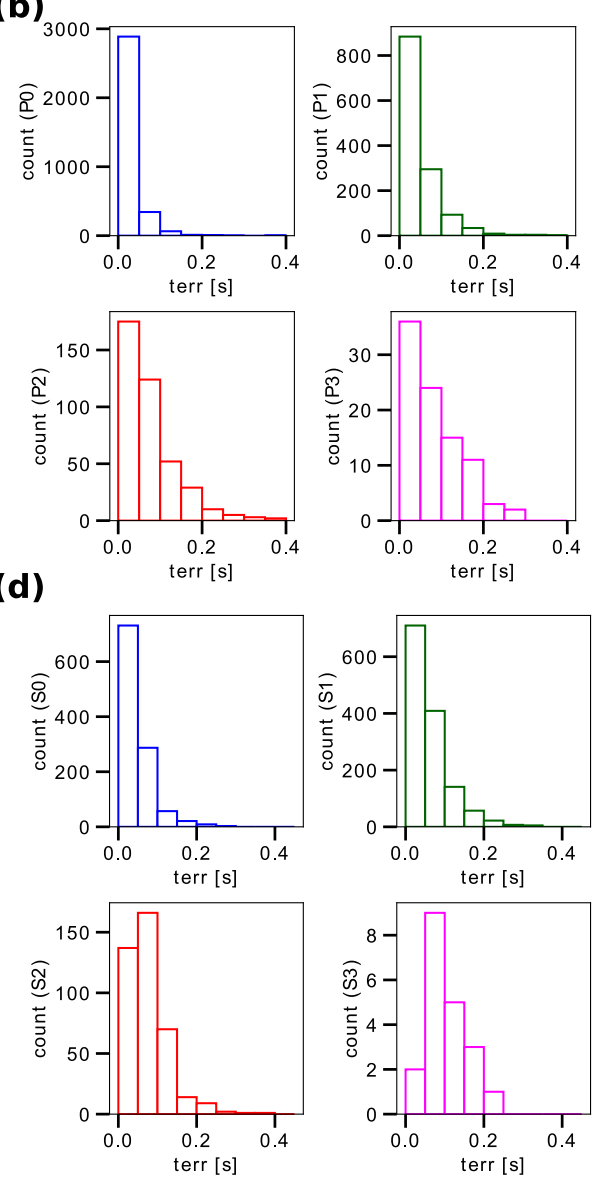

Figure 14. (a), (c) Normalized distribution of analyst P- and S-pick quality classes over the range of predicted time onset errors (terr in Table 3). Time errors were predicted on the augmented test sets, so analyst quality classes are counted twice. Points defining each colored curve are plotted at the center of the histogram bins corresponding to each pick class distribution. Dotted vertical black lines mark the time confidence interval limits used in the weighting scheme to assign the predicted pick classes (Table 3). (b), (d) Histograms (bin size $=0.05[\mathrm{~s}]$ ) showing disaggregated distributions of analyst P- and S-pick classes shown in (a) and (c), respectively.

Overall, however, the time residual distributions for both $\mathrm{P}$ and $\mathrm{S}$ phases show a remarkable compliance with analyst picks. Based on the weighting class scheme defined in Table 3, we observe that a significant majority of P- and S-phase onsets were predicted with lower uncertainties (pick quality classes 0 or 1 in Figs $12 \mathrm{~d}$ and $13 \mathrm{~d}$ ). Even though the distributions of all pick quality classes span over a similar range of time residuals, the distribution is more flattened for higher uncertainties. This may suggest that picks predicted with lower uncertainties by the network were also easier to pick by the analyst, resulting in lower time residuals since both the network and analyst would 
have picked a similar onset. Conversely, picks that were harder to pick by the analyst (e.g., due to a low SNR), were probably predicted with higher uncertainties by the network, which would lead to higher absolute residuals.

So as to investigate this correlation between predicted and analyst pick classes, we plotted the distribution of the analyst pick classes against the predicted time onset errors (terr) for both test sets (Fig. 14). Essentially, these distributions show that analyst classes do not always correlate with equivalent predicted classes, but instead span the entire range of predicted time onset errors.

However, a couple of observations from the normalized distributions in Figs 14(a) and (c) may indicate that at least some degree of correlation does exist. First, lower-uncertainty analyst picks (classes 0 and 1 ) concentrate in predicted lower-error intervals $($ terr $<0.05)$ in a considerably higher proportion, compared with higher-uncertainty analyst picks (classes 2 and 3). Second, analyst picks of higher uncertainty decrease more gradually and rise above picks of lower uncertainty when terr $>0.05$.

\subsection{Evaluation on independent test sets from automatically-derived earthquake catalogs}

We further analyzed the performance of the best trained models of DeepPhasePick on two additional independent test sets. These test sets consisted of three-component window samples extracted from two earthquake catalogs recently published for the region where the 2014 M8.1 Iquique megathrust earthquake ruptured the northern Chilean subduction on 1 April 2014 (Fig. 15). The first catalog (T1) contains 8,278 events occurring in a time window of nine months before the Iquique mainshock (between 2013-06-01 and 2014-02-28) (Sippl et al. 2018), from which we extracted 228,230 records (P: 88,449; N: 88,449; S: 51,332). The second catalog (T2) includes 18,963 events originating between one month before and nine months after the mainshock (between 2014-03-01 and 2014-12-31) (Soto et al.2019), from which we obtained 1,361,544 records (P: 545,746, N: 545,746, S: 270,052). Event records extracted from T1 and T2 were automatically picked by applying conventional state-of-the-art phase picking algorithms (Aldersons 2004; Di Stefano et al. 2006; Diehl et al. 2009), thus provide a valuable quantitative assessment when compared to DeepPhasePick predictions. 


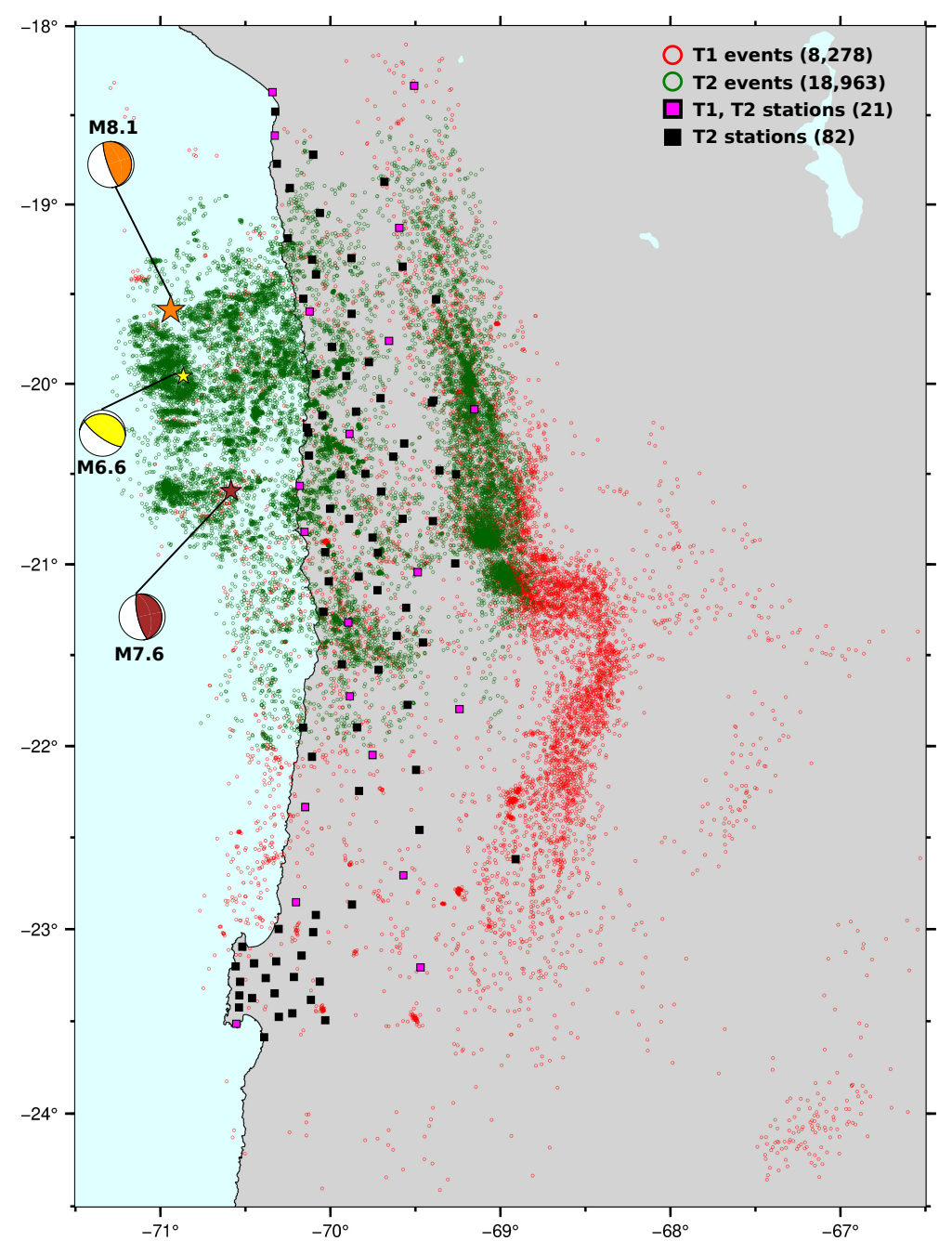

Figure 15. Automatically-derived earthquake catalogs in northern Chile, from which two independent test sets of three-component samples were used to evaluate the DeepPhasePick performance. A first test set of 228,230 samples was extracted from events occurring between 2013-06-01 and 2014-02-28 (T1: red circles; Sippl et al. 2018). A second test set of 1,361,544 samples was taken from events occurring between 201403-01 and 2014-12-31 (T2: green circles; Soto et al.|2019). Window samples obtained from T1 and T2 were registered by the 21 and 82 stations shown on the map. Focal mechanisms (beachballs) and epicenters (stars) of the Iquique M8.1 mainshock, its largest M6.6 foreshock and its largest M7.6 aftershock (Hayes et al. 2014) are plotted for reference in orange, yellow, and brown color, respectively.

We first analyzed the performance of the optimized model trained for phase detection. For this analysis, we used test sets formed by the entire set of samples available from catalogs T1 and T2. Fig. 16 summarizes the classification results from the evaluation of the model on both test sets. In the confusion matrices in Figs 16(a) and (c), we observe the highest ratio of correct predictions for noise samples, comparable to the evaluation performed on the test set extracted from datasets S1 
(a)

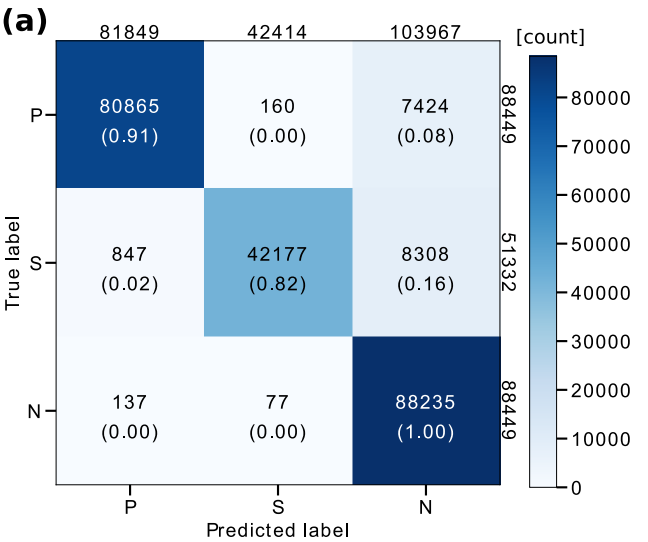

(c)

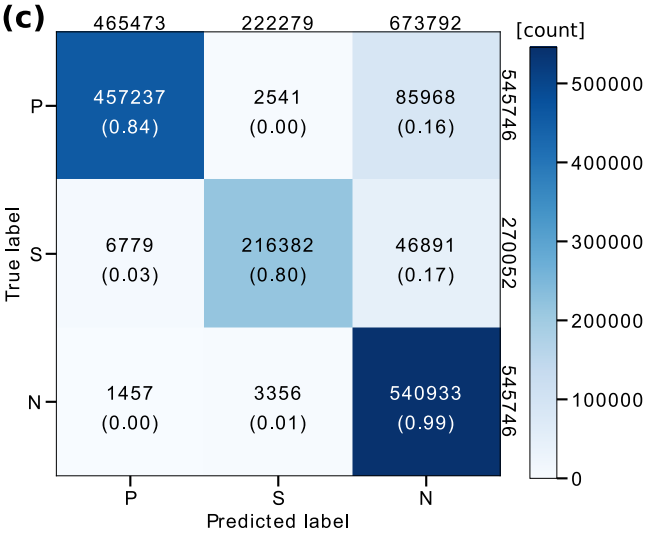

(b)
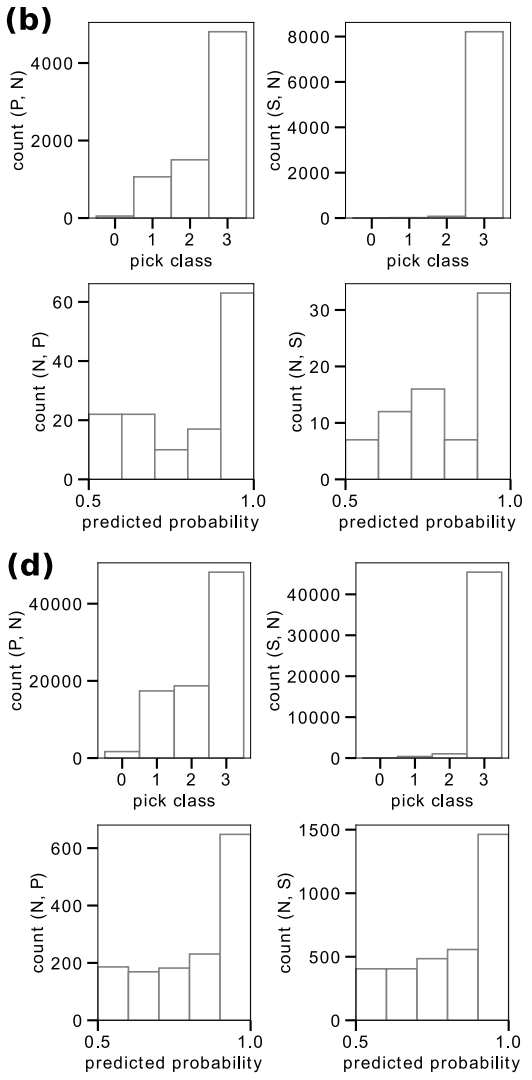

Figure 16. Results from the evaluation of the best model trained for phase detection on independent test sets taken from automatically-derived earthquake catalogs T1 (a-b) and T2 (c-d). (a), (c) Confusion matrices. (b), (d) disaggregated histograms of incorrectly predicted $\mathrm{P}$ or S phases as noise (upper subplots) and presumably misclassified noise samples as $\mathrm{P}$ or $\mathrm{S}$ phases (lower subplots). (P, N) means P-phase samples predicted as Noise, and similarly for other cells in the confusion matrix.

and S2 (Fig. 7c). The percentage of correct classifications decreased by $8-15 \%$ for P samples and $17-19 \%$ for S samples.

From the mispredicted $\mathrm{P}$ and $\mathrm{S}$ records, only a small fraction corresponds to $\mathrm{P}$ phase mispredicted as $\mathrm{S}$, or viceversa, compared to the number of $\mathrm{P}$ and $\mathrm{S}$ records which were predicted as noise. As we observed for the results in Fig. 7(c), the optimized model can mispredict S phases as $\mathrm{P}$ phases if the true $\mathrm{S}$ time onsets are somewhat earlier. Conversely, it is conceivable that the network classifies a $\mathrm{P}$ phase as an $\mathrm{S}$ phase if the true $\mathrm{P}$ time onset is late.

$\mathrm{P}$ and $\mathrm{S}$ phases predicted as noise correspond almost entirely to samples which were assigned automatic pick quality class 1 or worse ( $<75 \%$ confidence), with most of them being assigned the poorest pick quality class (class $3 ;<25 \%$ confidence) (upper subplots in Figs $16 \mathrm{~b}$ and d). As we 

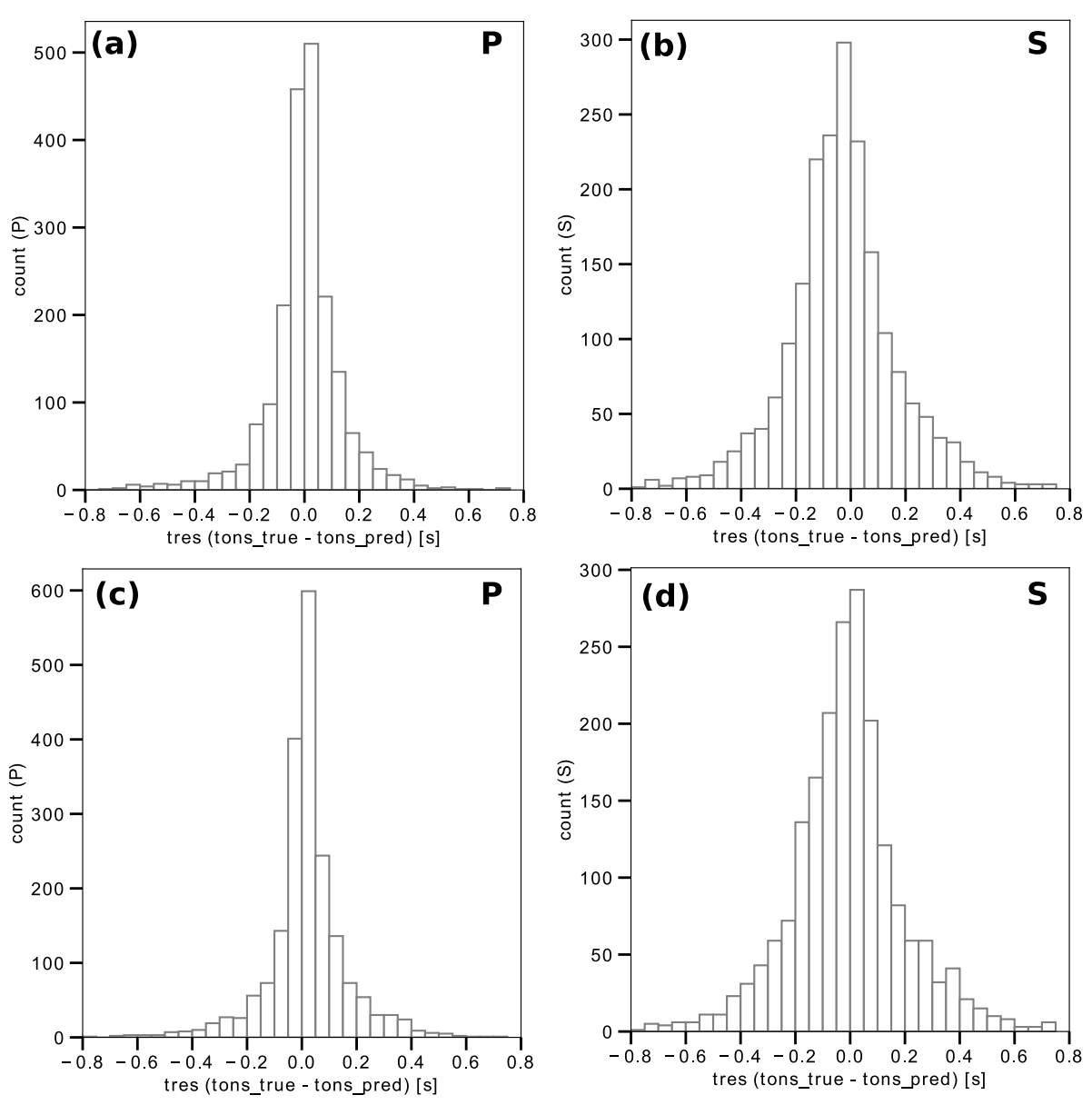

Figure 17. Results from the evaluation of the optimized model trained for P-phase picking on independent augmented test sets formed by $1,000 \times 2$ samples taken from the automatically-derived earthquake catalogs T1 (a-b) and T2 (c-d). (a) P-phase time residuals distribution for test samples taken from T1 (as in Fig. 12k). (b) S-phase time residuals distribution for test samples taken from T1 (as in Fig. 13k). (c) P-phase time residuals distribution for test samples taken from T2. (b) S-phase time residuals distribution for test samples taken from $\mathrm{T} 2$.

have discussed, the classification of these hard-to-pick phases by the network appears to be challenging. Moreover, about half of the few apparently misclassified noise samples were predicted as $\mathrm{P}$ or $\mathrm{S}$ phases with a probability of at least $90 \%$ (lower subplots in Figs $16 \mathrm{p}$ and d), suggesting that these presumed noise records might actually represent phases previously undetected by the automatic detection procedure, similar to the examples shown in Fig. 9 .

We next analyzed the performance of the optimized models trained for P- and S-phase picking, using test sets formed by 1,000 P- and S-phase samples randomly selected from T1 and T2 

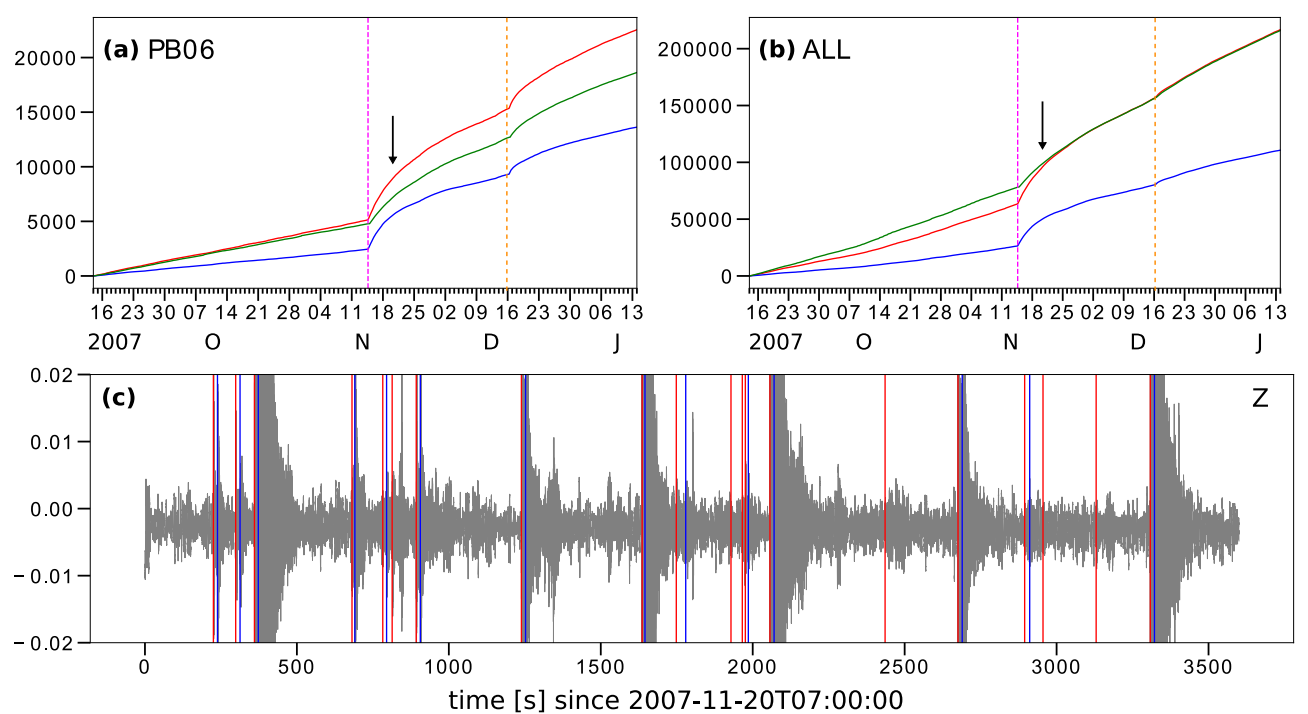

Figure 18. (a), (b) Cumulative number of $P$ (red curve) and $S$ (blue curve) phases predicted by DeepPhasePick $\left(p b_{-} P \_t h 1=p b_{-} S \_t h 1=0.98\right)$, and triggered detections by the STA/LTA algorithm (green curve) on continuous seismic data recorded at the PB06 station (a) and across all IPOC stations with available data (b). Cumulative curves are plotted for the time interval covering two months before up to two months after the 2007 M7.7 Tocopilla mainshock (2007-11-14), which is marked as a magenta dashed line. An orange dashed line indicates the time of occurrence of a M7.1 aftershock (2007-12-16) 50 km south of the mainshock epicenter. (c) One-hour waveform snippet of PB06 station data, depicting the phases predicted by DeepPhasePick $\left(p b_{-} P \_t h 1=p b_{-} S_{-} t h 1=0.98\right)$ at the time indicated by the arrows in $(\mathrm{a})$ and $(\mathrm{b})$ : red and blue lines represent $\mathrm{P}$ and $\mathrm{S}$ phases, respectively.

catalogs. As described in the previous section, the picking models were evaluated on augmented versions of these test sets.

Results from the picking predictions are summarized in Fig. 17. Time residual distributions, which are narrower for $\mathrm{P}$ phases (Figs $17 \mathrm{a}$ and c) compared to S phases (Figs 17b and d), are observed in both test sets. Similar distribution shapes were obtained for the augmented test set derived from S1 and S2 datasets (Figs $12 \mathrm{k}$ and $13 \mathrm{c}$ ), hence evidencing that the trained picking models generalize effectively to different datasets.

\subsection{Prediction on continuous seismic waveform data}

After analyzing the performance of DeepPhasePick with different independent sets, we used the best-performing trained models in the algorithm to carry out the phase detection and picking tasks on continuous seismic data recorded in two different tectonic environments. First, we 

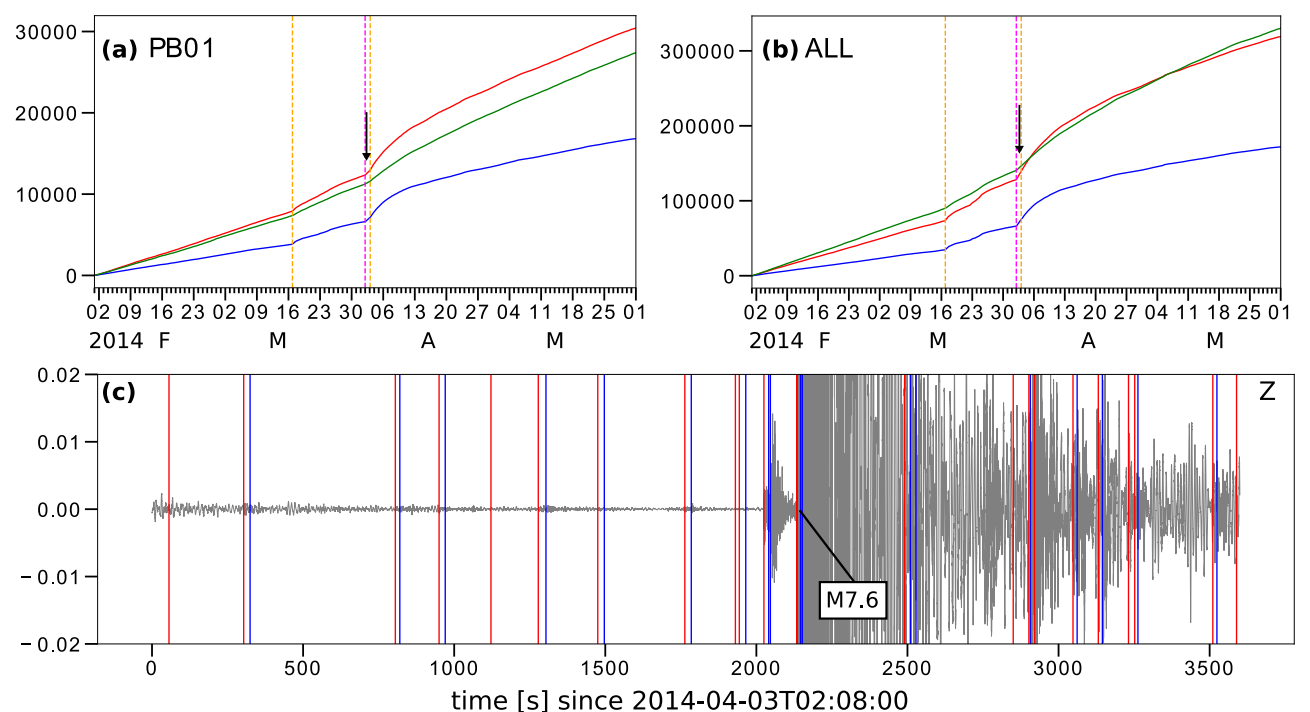

Figure 19. (a), (b) Cumulative number of $P$ (red curve) and $S$ (blue curve) phases predicted by DeepPhasePick $\left(p b_{-} P_{-} t h 1=p b_{-} S_{-} t h 1=0.98\right)$, and triggered detections by the STA/LTA algorithm (green curve) on continuous seismic data recorded at the PB01 station (a) and across all IPOC stations with available data (b). Cumulative curves are plotted for the time interval covering two months before up to two months after the 2014 M8.1 Iquique mainshock (2014-04-01), which is marked as a magenta dashed line. Two additional orange dashed lines indicate the time of occurrence of the largest foreshock (M6.7; 2014-03-16) and aftershock (M7.6; 2014-04-03) in the Iquique sequence. (c) One-hour waveform snippet of PB01 station data, depicting the phases predicted by DeepPhasePick $\left(p b_{-} P_{-} t h 1=p b_{-} S_{-} t h 1=0.95\right)$ at the time indicated by the arrows in (a) and (b): red and blue lines represent $\mathrm{P}$ and $\mathrm{S}$ phases, respectively.

applied DeepPhasePick on data which covers the time of two megathrust earthquake sequences that occurred in the northern Chile subduction zone (2007-11-14 M7.7 Tocopilla and 2014-04-01 M8.1 Iquique mainshocks). In this case, the data used was recorded by network stations situated in desertic areas, far from cities, and therefore exposed to low background seismic noise. Next, DeepPhasePick was applied on seismic data that partly covers the aftershock series of a recent M6.4 earthquake which occurred on 2019-11-26 near the port town in Durres, Albania, in a region of convergence between Adriatic and Eurasian plates. Here we used seismic data from a 30-station network deployed in the rupture area of the M6.4 earthquake, about one month after its occurrence. This station network covers an urban area, thus being subject to higher seismic-noise signals compared to northern Chile.

We numerically compared the prediction performance of DeepPhasePick in both tectonic regimes with the detections done by an in-house developed STA/LTA trigger algorithm applied 

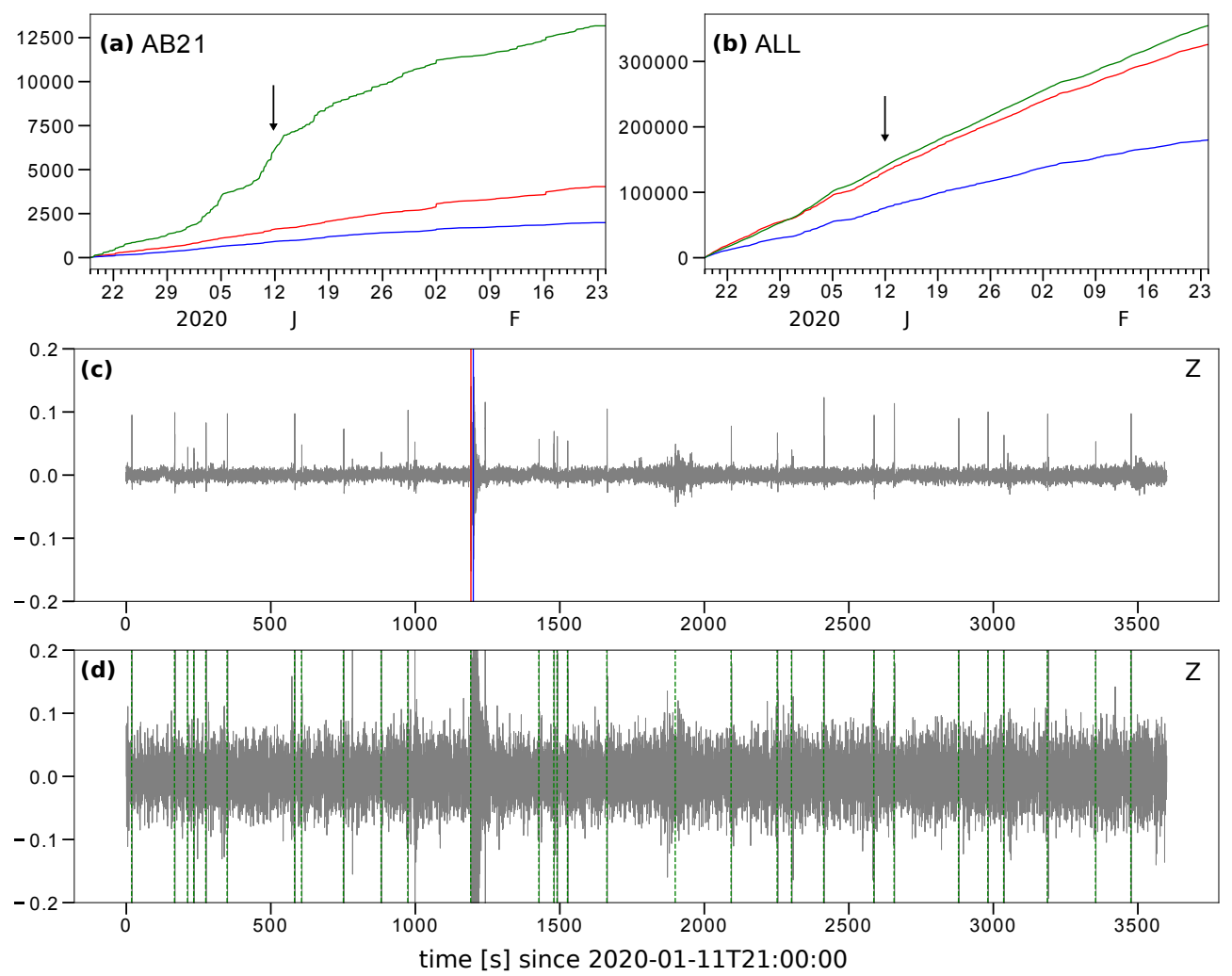

Figure 20. (a), (b) Cumulative number of $P$ (red curve) and $S$ (blue curve) phases predicted by DeepPhasePick $\left(p b \_P \_t h 1=p b \_S \_t h 1=0.98\right)$, and triggered detections by the STA/LTA algorithm (green curve) on continuous seismic data recorded at the AB21 station (a) and across all Albanian stations with available data (b). Cumulative curves are plotted for the time interval between 2019-12-19 and 2020-02-24, during the aftershock sequence of the 2019 M6.4 Albania earthquake (2019-11-26). (c) One-hour waveform snippet of AB21 station data, depicting the phases predicted by DeepPhasePick $\left(p b_{-} P \_t h 1=p b_{\_} S \_t h 1=0.98\right)$ at the time indicated by the arrows in (a) and (b): red and blue lines represent $\mathrm{P}$ and $\mathrm{S}$ phases, respectively. (d) Green dashed lines depict the STA/LTA detections produced on the one-hour waveform plotted in (c).

to all vertical component waveforms. For this test, we used STA and LTA window lengths of 1.0 and $20.0 \mathrm{~s}$; STA/LTA trigger and detrigger ratios of 8.0 and 1.5; and bandpass filter lower and upper corner frequencies of 2.0 and $10.0 \mathrm{~Hz}$.

\subsubsection{Performance in a lower-seismic noise region: northern Chile subduction zone}

The cumulative number of $\mathrm{P}$ and $\mathrm{S}$ phases predicted by DeepPhasePick on data from one representative as well as all available stations in northern Chile, depict an abrupt increase right after the occurrence of the 2007 M7.7 Tocopilla mainshock (Figs 18 and b) and the 2014 M8.1 Iquique mainshock (Figs 19 a and b), corresponding to the beginning of the aftershock series of these large 


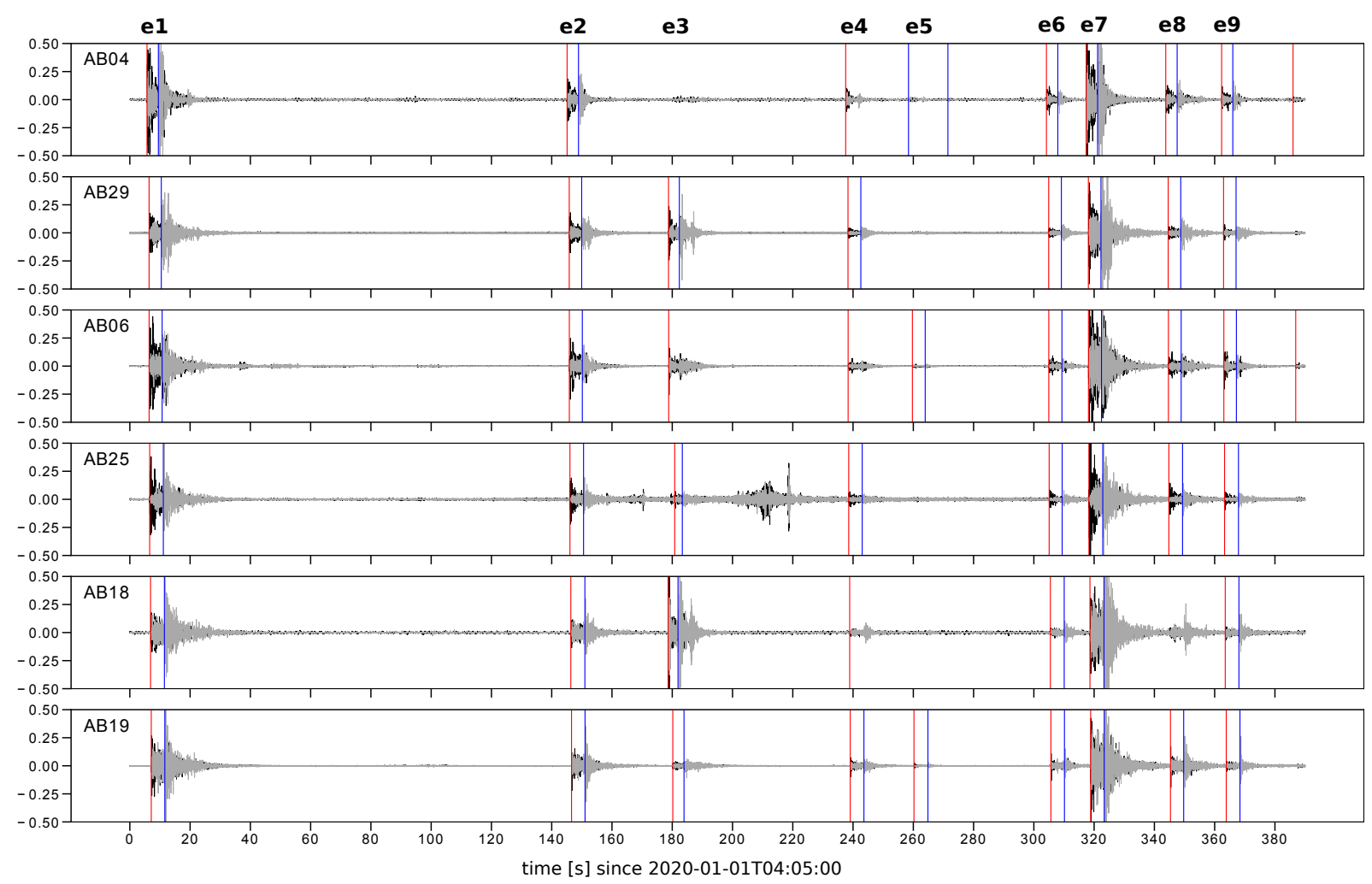

Figure 21. Example of multi-station phase prediction made by DeepPhasePick $\left(p b_{-} P \_t h 1=p b_{-} S_{-} t h 1=\right.$ 0.98) on continuous seismic data in Albania. Red and blue lines mark predicted P and S phase time onsets, respectively. Horizontal north component (gray) is plotted on top of vertical component (black) in each station waveform. Several events are clearly detected across the station network.

megathrust earthquakes. Less pronounced increments in cumulative predictions are observed at other times as well: after a M7.1 aftershock occurring approximately one month following the Tocopilla mainshock (Figs $18 \mathrm{a}$ and b), and after the occurrence of the largest foreshock (M6.7; 2014-03-16) and aftershock (M7.6; 2014-04-03) of the Iquique sequence (Figs 19a and b).

DeepPhasePick successfully captured a large majority of the various sized events occurring in the early postseismic stage of the Tocopilla and Iquique mainshocks (Figs 18c and 19k), which demonstrates the high resolution power of the algorithm. In particular, DeepPhasePick was capable of detecting $\mathrm{P}$ and $\mathrm{S}$ phases of several events occurring only a few minutes after the M7.6 aftershock in the Iquique earthquake sequence (Fig. 19k). 


\subsubsection{Performance in a higher-seismic noise region: Albania}

For most of the stations in northern Chile, the cumulative number of $\mathrm{P}$ phases predicted by DeepPhasePick is comparable to the cumulative detections made by a classical STA/LTA algorithm (Figs S3 and S4), as one might expect from high-SNR seismic data. However, this does not hold true for several stations in Albania (Figs S5 and S6), which presumably recorded lower-SNR seismic data, though the overall cumulative number of predicted P phases and STA/LTA detections across all the stations in this region is still comparable (Fig. 20p).

The discrepancy between the cumulative STA/LTA detections and DeepPhasePick predictions in the Albanian data arises mostly in two distinguishable scenarios. In the first scenario, cumulative STA/LTA detections greatly surpass P- and S-phase predictions (e.g., stations AB05, AB12, or AB21). Figs 20(c) and (d) illustrate one example of this, where the STA/LTA algorithm detects numerous false positives in the noisy one-hour waveform, whereas DeepPhasePick predicts only one P- and S-phase occurrence. In the second scenario, cumulative P-phase predictions are considerably higher than STA/LTA detections (e.g., stations AB10 or AB27) (Figs S5 and S6). In this case, presumed false positive predicted by DeepPhasePick could be discarded, for instance, if they are not detected in a minimum number of stations when applying a phase associator algorithm.

In spite of eventual mispredictions generated due to noisy data, DeepPhasePick is generally able to detect multiple events across a station network. One example of multi-station prediction is shown in Fig. 21, where six events are well detected in at least five stations within a 400s window. Fig. 22 shows the corresponding time onsets for one of these events, generated by applying the MCD method in the picking stage. Similar results for two more events shown in Fig. 21 are presented in Figs S7 and S8. From the statistics of the picks predicted for the three events shown in Figs 22, S7, and S8, which are reported in Tables S1, S2 and S3, we observe that the difference between the preliminary phase time onsets (tons_prelim) obtained in the phase detection stage and the refined ones (tons_pred) computed in the picking stage can be up to 0.13 [s] for P phases and up to 0.17 [s] for S phases. 

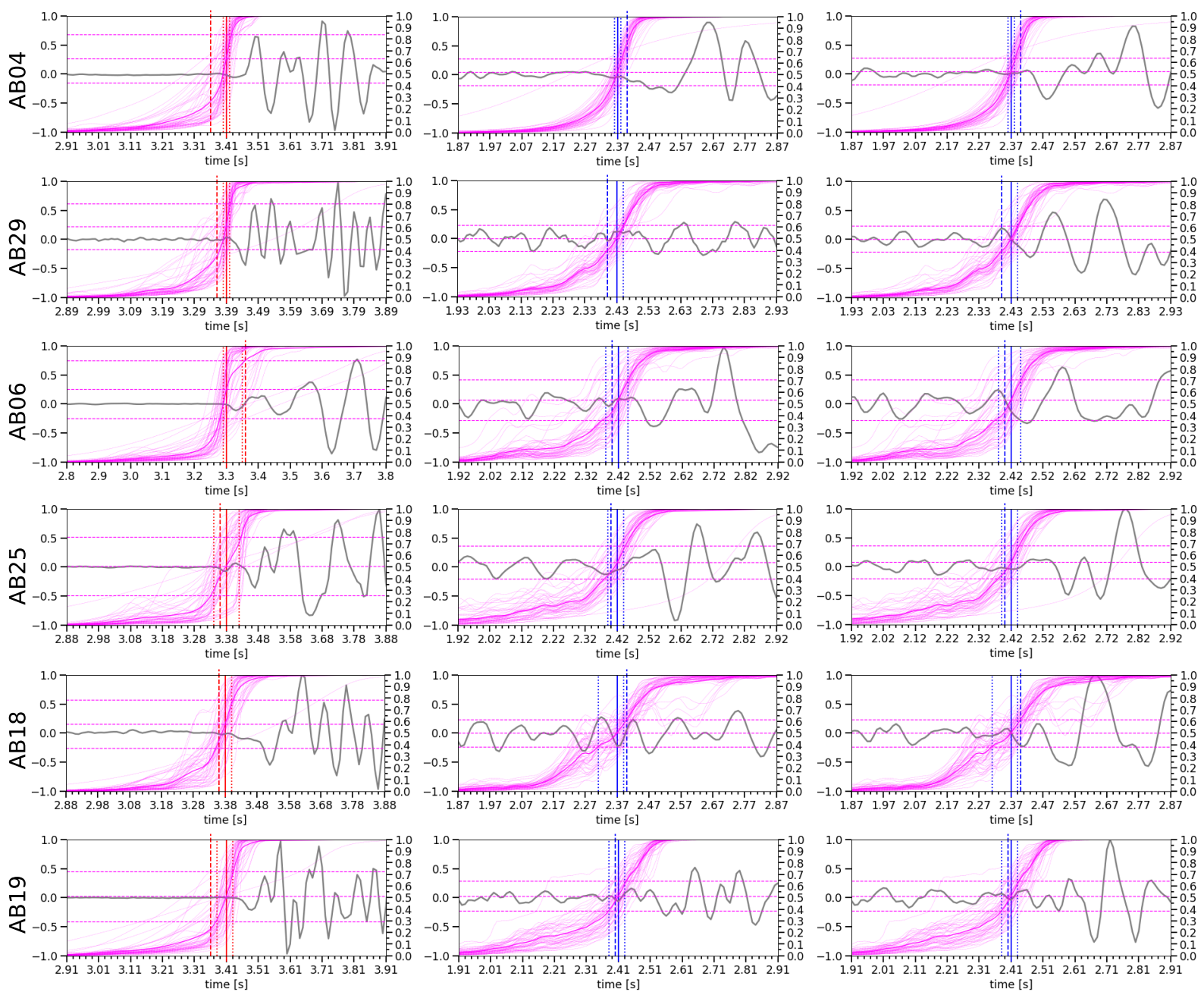

Figure 22. Estimation of time onset and associated uncertainty for predicted $\mathrm{P}$ (left) and $\mathrm{S}$ (center: E component, left: $\mathrm{N}$ component) phases of the event $\mathbf{e} 1$ in Fig. 21. Results from applying MCD method to each seismogram component are shown as in Fig. 3 . Pick statistics for this event are reported in Table S1.

\section{DISCUSSION}

We have demonstrated that DeepPhasePick can be successfully applied to accurately detect and pick $\mathrm{P}$ and $\mathrm{S}$ phases originating from local earthquakes. The accurate predictions produced by DeepPhasePick result from the highly optimized set of hyperparameters defining its convolutional and recurrent deep neural networks trained for the tasks of seismic phase detection and picking, respectively. The systematic optimization process implemented aimed at compensating the limited seismic data used for training the models.

Results from the phase detection model optimization give us clues on the network architecture as well as input seismic samples characteristics that make the model perform more efficiently. 
Firstly, the optimization indicates that the network detection capacity is enhanced by using a deeper block of convolutional layers. This can be explained by the fact that the model's ability to learn patterns from the input data, as well as the model's capacity to generalize so as to identify those patterns in new unseen data, are usually enhanced by adding convolutional layers to a CNN-based model. A large number of dense layers in the network, which aim at learning non-local relationships in the input data, does not seem to be required for improving network performance. Secondly, the network learns features in $\mathrm{P}$ and $\mathrm{S}$ phases more effectively when it is fed with relatively long input seismic windows which have not been filtered. Longer windows may ease the extraction of relevant seismic features in order to better discriminate between $\mathrm{P}$ and $\mathrm{S}$ phases and identify noise, particularly if very long-period background seismic noise is present. Interestingly, the network detects $\mathrm{P}$ phases better when sample windows contain a larger fraction of noise prior to a shorter portion of signal, in a way that resembles how analysts commonly identify this phase. Unlike P phases, optimized detection of $\mathrm{S}$ phases is obtained on sample windows consisting of a similar fraction of noise relative to signal, which may imply that the model learns patterns from features such as the $\mathrm{S}$ coda in order to recognize this phase.

Predictions performed on seismic samples from two independent test sets show that DeepPhasePick is capable of recognizing manually as well as automatically picked $\mathrm{P}$ and $\mathrm{S}$ phases with high accuracy, although it decreases for lower-quality automatic picks. These results also demonstrate that DeepPhasePick predicts phase time onsets which are comparable to those picked by analysts, as can be seen from the narrow time residual distributions in Figs 12 (c) and 13.c). These residuals are comparable to those obtained in previous studies that implemented a deep learningbased picking approach (Ross et al. 2018a; Zhu \& Beroza 2018), even though a much smaller dataset was used for training DeepPhasePick. Moreover, some degree of correlation between predicted and analyst pick classes was found (see Fig. 14). We note here that phase-pick weighting made by analysts is not an exact measure, but usually based on experience and intuition. Hence we would not necessarily expect a perfect correlation, and the weighting provided by DeepPhasePick might in fact be more objective.

In particular, we have shown that DeepPhasePick predicts P-and S-phase time onsets which are 
at least comparable to the time onsets obtained by applying dedicated automatic picking algorithms such as MPX (Aldersons 2004; Di Stefano et al. 2006) and Spicker (Diehl et al. 2009), which served to derive the high resolution catalogs for the Northern Chile region shown in Fig. 15. Since DeepPhasePick also computes uncertainties and weights associated to the predicted picks, it can be used as a compelling alternative to those methods.

However, DeepPhasePick is not restricted for use in the northern Chilean subduction zone, where the seismic samples used in training the models were originated. The algorithm is also able to predict $\mathrm{P}$ and $\mathrm{S}$ phases from local earthquakes occurring in a different tectonic regime, as we demonstrated for seismic data recorded by a 30-station network in a region in Albania (e.g., Fig. 21].

DeepPhasePick has been designed to internally share the knowledge learned from the optimization of the phase detection model to the phase picking models, allowing the algorithm to perform both tasks in a joint two-stage process. Consequently, DeepPhasePick can be applied directly on continuous seismic waveforms with minor preprocessing involved, so as to determine accurate $\mathrm{P}$ and $\mathrm{S}$ time arrivals that can then feed a phase associator algorithm in the next stage of an automatic earthquake location workflow.

Further improvement of DeepPhasePick can be addressed in future work, for instance by retraining the $\mathrm{CNN}$-based phase detection model with additional manually-picked samples from a new region of interest. A simple way to achieve this upgrade would be to use the new data for applying techniques such as feature extraction and fine tuning on the optimized model here presented. Further, the RNN-based phase picking models can be retrained by using more than one shifted version of each available seismic record (see e.g., Ross et al.|2018a). This would provide a broader spectrum of learnable sequential data during the training, which might improve the picking models performance, though with the caveat that the computing time required to train the models would further increase. 


\section{CONCLUSION}

The fast development in deep learning algorithms has made it possible to reach super-human performance levels in tasks involving huge amount of data, such as image classification and natural language processing. In this work, we leveraged this computational progress to develop DeepPhasePick, a novel two-stage algorithm for detection and picking of $\mathrm{P}$ and $\mathrm{S}$ seismic phases originating from local earthquakes.

DeepPhasePick has been built based on highly optimized convolutional and recurrent deep neural network architectures trained for such tasks. In a first stage, DeepPhasePick reports probabilities of waveform samples belonging to three phase classes (P, S or Noise). Preliminary phase onsets obtained from these probabilities are refined in a second step, in which final time onsets and their associated uncertainty are obtained by applying the Monte Carlo Dropout regularization technique, as an approximation of Bayesian variational inference.

DeepPhasePick has proven capable of recognizing both manually and automatically picked $\mathrm{P}$ and S seismic phases with high accuracy. It can also predict phase time onsets, which are comparable to those picked by analysts or derived from conventional, dedicated automatic phase picking algorithms. The P- and S-phase time onsets, as well as their associated uncertainties, predicted by DeepPhasePick from continuous seismic data can be directly used to feed a phase associator algorithm as part of an automatic earthquake location workflow.

\section{ACKNOWLEDGMENTS}

H. S. was previously supported by a CONICYT-DAAD stipend (57144001) and currently by the HAzard and Risk Team (HART) initiative of the GFZ German Research Centre for Geosciences in collaboration with the Institute of GeoSciences, Energy, Water and Environment of the Polytechnic University Tirana, Albania and the Karlsruhe Institute of Technology, Germany. We thank the developers of Hyperopt library, as well as Tensorflow and Keras machine learning frameworks, on which DeepPhasePick architecture was built. We further thank the developers of Python packages Obspy (Beyreuther et al.2010) and Matplotlib (Hunter 2007), as well as Generic 
Mapping Tools (Wessel et al. 2019), which were used to create the figures and maps presented in this work.

\section{DATA AVAILABILITY}

For training DeepPhasePick models for phase detection and picking tasks, we used waveform earthquake data collected from networks ZB (Schurr et al. 1999), ZE (Haberland et al. 1996), 8F (Wigger et al. 2016), Y9 (Fuenzalida et al. 2013), CX (GFZ German Research Centre for Geosciences \& Institut des Sciences de l'Univers-Centre National de la Recherche CNRS-INSU|2006), and GE (GEOFON Data Centre 1993) accessed via EIDA web services (e.g., https://geofon.gfzpotsdam.de/).

For the evaluation of the performance of DeepPhasePick in independent test sets built from automatically-derived earthquake catalogs, we used waveform earthquake data taken from networks CX, IQ (Cesca et al.|2009), 3D (Asch et al.|2014), and GE, as well as from Chilean Seismological Network (C, C1) stations (Barrientos 2018) accessed via IRIS webservices (http://ds.iris.edu/ SeismiQuery/), the MEJIPE temporary network deployed by FU Berlin (Salazar et al. 2013) accessed via EIDA web services, and a temporary network deployed by the Chilean ONEMI, DGF, and CSN institutions accessed from CSN upon request.

The performance of DeepPhasePick predictions was further evaluated on continuous waveform data taken from networks CX and 9K (obtained from GEOFON data centre).

The optimized DeepPhasePick models trained for phase detection and phase picking tasks, together with an example script that applies DeepPhasePick method for both tasks on continuous waveforms, will be available through the GFZ Data Services at https://dataservices.gfzpotsdam.de/.

\section{REFERENCES}

Abadi, M., Agarwal, A., Barham, P., Brevdo, E., Chen, Z., Citro, C., et al., 2015. TensorFlow: Largescale machine learning on heterogeneous systems. Retrieved from https://www.tensorflow.org/ (software available from tensorflow.org). 
Aldersons, F. 2004. Toward a three-dimensional crustal structure of the dead sea region from local earthquake tomography, PhD thesis, Israel: Tel-Aviv University.

Allen R.V. 1978. Automatic earthquake recognition and timing from single traces, Bull. seism. Soc. Am., 68(5), 1521-1532.

Asch, G., Tilmann, F., Heit, B. \& Schurr, B., 2014. HART-PISAGUA Project Chile, GFZ Data Services. Other/Seismic Network. https://doi.org/10.14470/8Q7569558037

Baer, M. \& Kradolfer, U., 1987. An automatic phase picker for local and teleseismic events, Bull. seism. Soc. Am., 77(4), 1437-1445.

Barrientos, S., 2018. The seismic network of Chile, Seism. Res. Lett., 89(2A), 467-474.

Bergstra, J., Bardenet, R., Bengio, Y. \& Kégl, B., 2011. Algorithms for hyper-parameter optimization, Proceedings of Advances in Neural Information Processing Systems 24, 2546-2554.

Bergstra J. \& Bengio, Y,. 2012. Random search for hyper-parameter optimization, Journal of Machine Learning Research, 13, 281-305.

Beyreuther, M., Barsch, R., Krischer, L., Megies, T., Behr, Y. \& Wassermann, J., 2010. Obspy: a Python toolbox for seismology, Seism. Res. Lett., 81(3), 530-533.

Bergstra, J., Yamins, D. \& Cox, D., 2013a. Making a science of model search: hyperparameter optimization in hundreds of dimensions for vision architectures, Proceedings of the International Conference on Machine Learning 30, 115-123.

Bergstra, J., Yamins, D. \& Cox, D., 2013b. Hyperopt: a Python library for optimizing the hyperparameters of machine learning algorithms, Proceedings of the Python in Science Conference 12, 13-20.

Cesca, S., Sobiesiak, M., Tassara, A., Olcay, M., Günther, E., Mikulla, S. \& Dahm, T., 2009. The Iquique Local Network and PicArray, GFZ Data Services. Other/Seismic Network. https://doi.org/10.14470/vd070092

Chollet, F., 2015. Keras. https://github.com/ fchollet/keras.

Chollet, F., 2017. Xception: Deep Learning With Depthwise Separable Convolutions, Proceedings of the IEEE Conference on Computer Vision and Pattern Recognition, 1251-1258.

Diehl, T., Deichmann, N., Kissling, E. \& Husen, S., 2009. Automatic S-wave picker for local earthquake tomography, Bull. seism. Soc. Am., 99(3), 1906-1920.

Di Stefano, R., Aldersons, F., Kissling, E., Baccheschi, P., Chiarabba, C. \& Giardini, D., 2006. Automatic seismic phase picking and consistent observation error assessment: Application to the Italian seismicity. Geophys. J. Int., Geophys. J Int., 165(1), 121-134. https://doi.org/10.1111/j.1365-246X.2005.02799.x

Dokht, R.M., Kao, H., Visser, R. \& Smith, B., 2019. Seismic event and phase detection using timefrequency representation and convolutional neural networks, Seism. Res. Lett., 90(2A), 481-490.

Earle, P.S. \& Shearer, P.M., 1994. Characterization of global seismograms using an automatic-picking algorithm, Bull. seism. Soc. Am., 84, 366-376. 
Folesky, J., Kummerow, J. \& Shapiro, S. A., 2018. Patterns of rupture directivity of subduction zone earthquakes in northern Chile, J. geophys. Res.: Solid Earth, 123, 10785-10796.

Fuenzalida, A., Schurr, B., Lancieri, M., Sobiesiak, M. \& Madariaga, R., 2013. High-resolution relocation and mechanism of aftershocks of the 2007 Tocopilla (Chile) earthquake, Geophys. J Int., 194(2), 12161228.

Gal, Y., \& Ghahramani, Z., 2016a. Dropout as a Bayesian approximation: Representing model uncertainty in deep learning, Proceedings of the International Conference on Machine Learning 33, 1651-1660.

Gal, Y. \& Ghahramani, Z., 2016b. A theoretically grounded application of dropout in recurrent neural networks, Proceedings of Advances in Neural Information Processing Systems 29, 1019-1027.

GEOFON Data Centre, 1993: GEOFON Seismic Network. Deutsches GeoForschungsZentrum GFZ, Other/Seismic Network. https://doi.org/10.14470/TR560404

GFZ German Research Centre for Geosciences \& Institut des Sciences de l'Univers-Centre National de la Recherche CNRS-INSU, 2006. IPOC Seismic Network. Integrated Plate boundary Observatory Chile IPOC, Other/Seismic Network. https://doi.org/10.14470/PK615318

Gibbons, S.J. \& Ringdal, F., 2006. The detection of low magnitude seismic events using array-based waveform correlation, Geophys. J Int., 165(1), 149-166.

Goodfellow, I., Bengio, Y. \& Courville, A., 2016. Deep learning (Vol. 1). MIT Press, Cambridge, Massachusetts.

Haberland, C., Rietbrock, A., Asch, G. \& Chong, G., 1996. The ANCORP Seismic Network, GFZ Data Services. Other/Seismic Network. https://doi:10.14470/MR6441682066.

Harris, D. B., 1991. A waveform correlation method for identifying quarry explosions, Bull. seism. Soc. Am., 81, 2395-2418.

Harris, D. B., 1997. Waveform correlation methods for identifying populations of calibration events, Proceedings of the 19th Annual Seismic Research Symposium on Monitoring a CTBT, September 23-25, 604-614.

Harris, D. B., 2001. Subspace Techniques for Detecting Repeating Events, poster, SSA 2001 Annual Meeting, San Francisco, CA, 18-20 April 2001, abstract in Seism. Res. Lett., 72(2), p245.

Harris, D. B., 2006. Subspace detectors: Theory, Tech. Rep. (Internal Report UCRL-TR-222758, Lawrence Livermore National Laboratory, 2006).

Harris, D. B. \& Dodge, D.A., 2011. An autonomous system for grouping events in a developing aftershock sequence, Bull. seism. Soc. Am., 101, 763-774.

Harris, D. B. \& Paik, T., 2006. Subspace Detectors: Efficient implementation, Tech. Rep., (Internal Report UCRL-TR-223177, Lawrence Livermore National Laboratory, 2006).

Hayes, G. P., Herman, M. W., Barnhart, W. D., Furlong, K. P., Riquelme, S., Benz, H. M., et al., 2014. Continuing megathrust earthquake potential in Chile after the 2014 Iquique earthquake, Nature, 512, 
295-298.

Hinton, G., Deng, L., Dahl, G. E., Mohamed, A., Jaitly, N., Senior, A., et al., 2012. Deep neural networks for acoustic modeling in speech recognition, IEEE Signal Processing Magazine, 29, 82-97.

Hinz, T., Navarro-Guerrero, N., Magg, S. \& Wermter, S., 2018. Speeding up the Hyperparameter Optimization of Deep Convolutional Neural Networks, International Journal of Computational Intelligence and Applications, 17(2), 1850008.

Hochreiter, S. \& Schmidhuber, J., 1997. Long short-term memory, Neural Computation, 9, 1735-1780.

Huang, H., Xu, W., Meng, L., Burgmann, R. \& Baez, J. C., 2017. Early aftershocks and afterslip surrounding the $2015 \mathrm{Mw} 8.4$ Illapel rupture, Earth Planet. Sci. Lett., 457, 282-291.

Hubel, D. H. \& Wiesel, T. N., 1959. Receptive fields of single neurons in the cat's striate cortex, J. Physiol., 148, 574-591.

Hubel, D. H. \& Wiesel, T. N., 1962. Receptive fields, binocular interaction, and functional architecture in the cat's visual cortex, J. Physiol., 160, 106-154.

Hunter, J. D., 2007. Matplotlib: a 2D graphics environment, Comput. Sci. Eng., 9, 90-95.

Igarashi, T., Matsuzawa, T. \& Hasegawa, A., 2003. Repeating earthquakes and interplate aseismic slip in the northeastern Japan subduction zone, J. geophys. Res., 108(B5), 2249.

Ioffe, S. \& Szegedy, C., 2015. Batch normalization: Accelerating deep network training by reducing internal covariate shift. arXiv:1502.03167.

Kaggle challenge, 2014. Higgs boson machine learning challenge. Kaggle https://www.kaggle.com/c/higgs-boson.

Kato, A. \& Igarashi, T., 2012. Regional extent of the large coseismic slip zone of the $2011 \mathrm{Mw} 9.0$ TohokuOki earthquake delineated by on-fault aftershocks, Geophys. Res. Lett., 39, L15301.

Kingma, D. P. \& Ba, J., 2014. Adam: A method for stochastic optimization, ArXiv:1412.6980 [Cs].. Retrieved from http://arxiv.org/abs/1412.6980.

Kriegerowski, M., Petersen, G.M., Vasyura-Bathke, H. \& Ohrnberger, M., 2019. A deep convolutional neural network for localization of clustered earthquakes based on multistation full waveforms, Seismol. Res. Lett., 90.

Krizhevsky, A., Sutskever, I. \& Hinton, G., 2012. ImageNet classification with deep convolutional neural networks, Proceedings of Advances in Neural Information Processing Systems 25, 1097-1105.

LeCun, Y., Bengio, Y. \& Hinton, G., 2015. Deep learning, Nature, 521(7553), 436-444.

LeCun, Y., Boser, B., Denker, J. S., Henderson, D., Howard, R. E., Hubbard, W., et al., 1990. Handwritten digit recognition with a back-propagation network, Proceedings of Advances in Neural Information Processing Systems 2, 396-404.

LeCun, Y., Bottou, L., Bengio, Y. \& Haffner, P., 1998. Gradient-based learning applied to document recognition, Proceedings of the IEEE, 86, 2278-2324. 
Maceira, M., Rowe, C.A., Beroza, G. \& Anderson, D., 2010. Identification of low-frequency earthquakes in non-volcanic tremor using the subspace detector method, Geophys. Res. Lett., 37, L06303.

McBrearty, I.W., Delorey, A.A. \& Johnson, P.A., 2019. Pairwise association of seismic arrivals with convolutional neural networks, Seismol. Res. Lett., 90(2A), 503-509.

Moseley, B., Markham, A. \& Nissen-Meyer, T., 2018. Fast approximate simulation of seismic waves with deep learning, arXiv:1807.06873.

Nadeau, R. M. \& Johnson, L. R., 1998. Seismological studies at Parkfield VI: Moment release rates and estimates of source parameters for small repeating earthquakes, Bull. seism. Soc. Am., , 88(3), 790-814.

Nair, V. \& Hinton, G., 2010. Rectified linear units improve restricted Boltzmann machines, Proceedings of the International Conference on Machine Learning 27, 807-814.

Panakkat, A. \& Adeli, H., 2009. Recurrent neural network for approximate earthquaketime and location prediction using multiple seismicity indicators, Computer-Aided Civil and Infrastructure Engineering, 24, 280-292.

Peng, Z. \& Zhao, P., 2009. Migration of early aftershocks following the 2004 Parkfield earthquake, Nat. Geosci., 2, 877-881.

Perol, T., Gharbi, M. \& Denolle, M., 2018. Convolutional neural network for earthquake detection and location, Sci. Adv., 4(2), e1700578.

Ross, Z. E., Meier, M.-A. \& Hauksson, E., 2018a. P wave arrival picking and first-motion polarity determination with deep learning, J. geophys. Res.: Solid Earth, 123(6), 5120-5129.

Ross, Z. E., Meier, M.-A., Hauksson, E. \& Heaton, T. H., 2018b. Generalized seismic phase detection with deep learning, Bull. seism. Soc. Am., 108(5A), 2894-2901.

Ross, Z. E., Trugman, D., Hauksson, E. \& Shearer, P.M., 2019a. Searching for hidden earthquakes in Southern California, Science, 364, 767-771.

Ross, Z. E., Yue, Y., Meier, M.-A., Hauksson, E. \& Heaton, T. H., 2019b. PhaseLink: A deep learning approach to seismic phase association, J. geophys. Res.: Solid Earth, 124, 856-869.

Rumelhart, D., Hinton, G. \& Williams, R., 1986. Learning representations by back-propagating errors, Nature, 323, 533-536.

Salazar, P., Wigger, P., Bloch, W., Asch, G., Shapiro, S. A. \& Kummerow, J., 2013. MEJIPE, GFZ Data Services. Other/Seismic Network. https://doi.org/10.7914/SN/8G_2013

Scharf, L.L. \& Friedlander, B., 1994. Matched Subspace Detectors, IEEE Transactions on Signal Processing, 42, 2146-2157.

Schurr, B., Asch, G., Rietbrock, A., Kind, R., Pardo, M., Heit, B. \& Monfret, T., 1999. Seismicity and average velocities beneath the Argentine Puna Plateau, Geophys. Res. Lett., 26(19), 3025-3028.

Schurr, B., Asch, G., Rosenau, M., Wang, R., Oncken, O., Barrientos, S., et al., 2012. The 2007 M7.7 Tocopilla northern Chile earthquake sequence: Implications for along-strike and downdip rupture seg- 
mentation and megathrust frictional behavior, J. geophys. Res., 117(B05305).

Schurr, B., Rietbrock, A., Asch, G., Kind, R. \& Oncken, O., 2006. Evidence for lithospheric detachment in the central Andes from local earthquake tomography, Tectonophysics, 415, 203-223.

Schuster, M. \& Paliwal, K. K., 1997. Bidirectional recurrent neural networks, IEEE Transactions on Signal Processing, 45(11), 2673-2681.

Shelly, D.R., Beroza, G.C. \& Ide, S., 2007. Non-volcanic tremor and low-frequency earthquake swarms, Nature, 446, 305-307.

Shoji, D., Noguchi, R., Otsuki, S. \& Hino, H., 2018. Classification of volcanic ash particles using a convolutional neural network and probability, Scientific Reports, 8(1), 8111-12.

Simard, D., Steinkraus, P. Y. \& Platt, J. C., 2003. Best practices for convolutional neural networks, Proceedings of International Conference on Document Analysis and Recognition 7, 958-963.

Sippl, C., Schurr, B., Asch, G. \& Kummerow, J., 2018. Seismicity structure of the Northern Chile forearc from $>100,000$ double-difference relocated hypocenters, J. geophys. Res.: Solid Earth, 123, 4063-4087.

Sippl, C., Schurr, B., Yuan, X., Mechie, J., Schneider, F. M., Gadoev, M., et al., 2013. Geometry of the Pamir-Hindu Kush intermediate-depth earthquake zone from local seismic data, J. geophys. Res.: Solid Earth, 118, 1438-1457.

Sleeman, R. \& van Eck, T., 1999. Robust automatic P-phase picking: an online implementation in the analysis of broadband seismogram recordings, Phys. Earth Planet. Inter., 113, 265-275.

Soto, H., Sippl, C., Schurr, B., Kummerow, J., Asch, G., Tilmann, F., et al., 2019. Probing the northern Chile megathrust with seismicity: the 2014 M8.1 iquique earthquake sequence, J. geophys. Res.: Solid Earth, 124, 12935-12954.

Srivastava, N., Hinton, G., Krizhevsky, A., Sutskever, I. \& Salakhutdinov, R., 2014. Dropout: a simple way to prevent neural networks from overfitting, Journal of Machine Learning Research, 15, 1929-1958.

Sutskever, I., Vinyals, O. \& Le, Q., 2014. Sequence to sequence learning with neural networks, Proceedings of Advances in Neural Information Processing Systems 27, 3104-3112.

Taigman, Y., Yang, M., Ranzato, M. \& Wolf, L., 2014. Deepface: closing the gap to human-level performance in face verification, Proceedings of the IEEE Conference on Computer Vision and Pattern Recognition, 1701-1708.

Van Trees, H.L., 1968. Detection, Estimation and Modulation Theory, John Wiley and Sons, Inc., New York.

Wessel, P., Luis, J., Uieda, L., Scharroo, R., Wobbe, F., Smith, W. H. F. \& Tian, D., 2019. The Generic Mapping Tools Version 6, Geochem. Geophys. Geosyst., 20 (11), 5556-5564.

Wigger, P., Salazar, P., Kummerow, J., Bloch, W., Asch, G. \& Shapiro, S., 2016. West-Fissure- and Atacama-Fault Seismic Network (2005/2012). Deutsches GeoForschungsZentrum, GFZ. Other/Seismic Network. https://doi:10.14470/3S7550699980. 


\section{H. Soto \& B. Schurr}

Woollam, J., Rietbrock, A., Bueno, A. \& Angelis, S. D., 2019. Convolutional neural network for seismic phase classification, performance demonstration over a local seismic network, Seismol. Res. Lett., 90(2A), 491-502.

Zhang, X., Zhang, J., Yuan, C., Liu, S., Chen, Z. \& Li, W., 2020. Locating induced earthquakes with a network of seismic stations in Oklahoma via a deep learning method, Scientific Reports, 10(1), 1-12.

Zhu, W. \& Beroza, G.C., 2018. Phasenet: a deep-neural-network-based seismic arrival-time picking method, Geophys. J Int., 216(1), 261-273. 


\title{
Supporting Information for
}

\section{DeepPhasePick: A method for Detecting and Picking Seismic}

Phases from Local Earthquakes based on highly optimized

\section{Convolutional and Recurrent Deep Neural Networks}

\author{
Hugo Soto and Bernd Schurr \\ German Research Centre for Geosciences (GFZ), Potsdam, Germany. E-mail: soto@gfz-potsdam.de
}

\section{CONTENTS}

1. Text $\mathrm{S} 1$

2. Figures $\mathrm{S} 1$ to $\mathrm{S} 8$

3. Tables $\mathrm{S} 1$ to $\mathrm{S} 3$

Text S1: Additional optional conditions for improving phase detection.

In order to enhance the performance of DeepPhasePick in correctly detecting P and S phases, we included some additional conditions to the procedure described in section 2.5 of the manuscript. These conditions apply criteria based on the P- and S-phase predicted probabilities in order to resolve some special cases, where discriminating between $\mathrm{P}$ and $\mathrm{S}$ phases is not trivial. The different conditions can be optionally activated by the user and depend on a few user-defined parameters, as described below.

(1) Resolve between $P$ and $S$ phases predicted close in time.

(i) For each predicted $\mathrm{P}$ onset $(t P)$, several threshold time intervals are defined as follows:

$$
\begin{aligned}
& \Delta t_{t h} \in\left[t P_{-} t h_{-} \text {pre }, t P_{-} t h_{-} \text {pos }\right], \\
& \Delta t_{t h \_p r e} \in\left[t P-d t_{-} P S_{-} m a x, t P_{-} t t_{-} p r e\right],
\end{aligned}
$$




$$
\Delta t_{t h \_p o s} \in\left[t P \_t h \_p o s, t P+d t \_P S \_m a x\right] .
$$

Here:

$$
\begin{aligned}
& t P_{-} t h_{-} p r e=t P-t\left(p b_{-} P \_p r e>0.5\right), \\
& t P_{-} t h_{-} p o s=t P+t\left(p b_{-} P_{-} p o s>0.5\right),
\end{aligned}
$$

where $t\left(p b_{-} P_{-} p r e>0.5\right)$ and $t\left(p b_{\_} P_{-} p o s>0.5\right)$ are the times at which the predicted probabilities before $\left(p b_{-} P \_p r e\right)$ and after $\left(p b_{-} P_{-}\right.$pos) $t P$ rise above 0.5 , and $d t_{-} P S \_\max [\mathrm{s}]$ is an user-defined parameter.

(ii) If a predicted $\mathrm{S}$ onset $(t S)$ is found at $t \in \Delta t_{t h}$, we resolve between $\mathrm{P}$ and $\mathrm{S}$ as follows:

- we keep $t P$ and discard $t S$, if

$\rightarrow p b_{-} P(t P) \geq p b_{-} S(t S)$,

$\rightarrow p b_{-} S(t S)>p b_{-} P(t P)$, but no $\mathrm{P}$ is found at $t \in \Delta t_{t h \_}$pre , and at least one $\mathrm{P}$ or $\mathrm{S}$ is found at $t \in \Delta t_{\text {th_pos }}$

- we keep $t S$ and discard $t P$, if

$\rightarrow p b_{-} S(t S)>p b_{-} P(t P)$, at least one $\mathrm{P}$ is found at $t \in \Delta t_{t h \_}$pre , and at least one $\mathrm{P}$ or $\mathrm{S}$ is found at $t \in \Delta t_{\text {th_pos }}$

$\rightarrow p b_{-} S(t S)>p b_{-} P(t P)$, at least one $\mathrm{P}$ is found at $t \in \Delta t_{t h \_p r e}$, and no $\mathrm{P}$ or $\mathrm{S}$ are found at $t \in \Delta t_{\text {th_pos }}$

- we discard both $t S$ and $t P$, if

$\rightarrow p b_{-} S(t S)>p b_{-} P(t P)$, but no $\mathrm{P}$ is found at $t \in \Delta t_{t h \_p r e}$, and no $\mathrm{P}$ or $\mathrm{S}$ are found at $t \in \Delta t_{t h \_p o s}$

(2) Discard predicted S phases for which there is no earlier P or P-S phases predicted.

(i) For each predicted $\mathrm{S}$ onset $(t S)$, the following threshold time intervals are defined:

$\Delta t_{\text {th_pre_1 }}^{S} \in\left[t S-d t_{-} P S_{-} \max \times d t_{-} P S_{-} f r a c, t S\right]$, where $d t_{-} P S_{-} f r a c$ is an user-defined parameter.

$$
\Delta t_{\text {th_pre_2 }}^{S} \in\left[t P_{-} \text {pre }, t S\right], \text { where } t P_{-} \text {pre } \in \Delta t_{\text {th_pre_1 }}^{S}
$$

(ii) We discard $t S$, if

$\rightarrow$ no $t P_{-}$pre $\in \Delta t_{\text {th_pre_1 }}^{S}$ is found.

$\rightarrow$ at least one $t P_{-}$pre $\in \Delta t_{t h \_p r e \_1}^{S}$ is found, but more than one $t S_{-}$pre $\in \Delta t_{t h \_p r e \_2}^{S}$ are found. 
$\rightarrow$ at least one $t P_{-} p r e \in \Delta t_{t h-p r e \_1}^{S}$ is found, one $t S_{-}$pre $\in \Delta t_{t h \_p r e \_2}^{S}$ is found, but $t S>t S_{-}$pre + $\left|t P \_p r e-t S \_p r e\right|$.

(3) Resolve between possible predicted duplicated S phases.

(i) For each predicted $\mathrm{S}$ onset $t S$, the following threshold time intervals are defined:

$$
\begin{aligned}
& t S_{-} t h_{-} p o s=t S+t\left(p b_{-} S_{-} p o s>0.5\right), \\
& t \hat{S}_{-} t h_{-} p r e=t \hat{S}-t\left(p b_{-} \hat{S}_{-} p r e>0.5\right),
\end{aligned}
$$

for each subsequent predicted $S$ onset $t \hat{S}(t \hat{S}>t S)$. Here $t\left(p b_{-} S_{-} p o s>0.5\right)$ and $t\left(p b_{-} \hat{S}_{-}\right.$pre $>$ $0.5)$ are the times at which the predicted probabilities after $t S\left(p b_{-} S_{-} p o s\right)$ and before $t \hat{S}$ ( $\left.p b_{-} \hat{S}_{-} p r e\right)$ rise above 0.5 , respectively.

(ii) If $\left|t S \_t h \_p o s-t \hat{S}_{-} t h \_p r e\right|<d t \_S d u p \_\max$, we only keep the $\mathrm{S}$ onset with the maximum predicted probability between $p b_{-} S(t S)$ and $p b_{-} \hat{S}(t \hat{S})$. Here $d t_{-} S d u p \_\max [\mathrm{s}]$ is an user-defined parameter.

(4) Resolve between $P$ and $S$ phases predicted close in time, for special cases not handled in (1).

(i) For each predicted $\mathrm{P}$ onset $(t P)$ and each subsequent predicted $\mathrm{S}$ onset $(t S)$, the following threshold time intervals are defined:

$$
\begin{aligned}
& t P \_t h \_p r e=t P-t\left(p b_{-} P \_p r e>0.5\right), \\
& t P \_t h \_p o s=t P+t\left(p b_{-} P \_p o s>0.5\right), \\
& t S_{-} t h \_p r e=t S-t\left(p b_{-} S_{-} p r e>0.5\right), \\
& t S_{-} t h \_p o s=t S+t\left(p b_{-} S_{-} p o s>0.5\right) .
\end{aligned}
$$

Here $t\left(p b_{-} P_{-}\right.$pre $\left.>0.5\right)$ and $t\left(p b_{-} P_{-}\right.$pos $\left.>0.5\right)$ are the times at which the predicted probabilities before $\left(p b_{-} P \_p r e\right)$ and after $\left(p b_{-} P \_p o s\right) t P$ rise above 0.5 . Similarly, $t\left(p b_{-} S \_p r e>0.5\right)$ and $t\left(p b_{-} S_{-} p o s>0.5\right)$ are the times at which the predicted probabilities before $\left(p b_{\_} S_{-} p r e\right)$ and after (pb_S_pos) $t S$ rise above 0.5 .

We also consider the threshold time differences between successive S-P or P-S predicted phases:

$$
\begin{aligned}
& d t_{-} S P_{-} t h=\left|t S_{-} t h_{-} p o s-t P_{-} t h_{-} p r e\right|, \\
& d t_{-} P S_{-} t h=\left|t P_{-} t h_{-} p o s-t S_{-} t h_{-} p r e\right|
\end{aligned}
$$




\section{$4 \quad$ H. Soto \& B. Schurr}

(ii) If $d t_{\_} S P_{\_} t h<d t_{-} S P_{\_} n e a r$ or $d t_{-} P S_{-} t h<d t_{-} S P \_n e a r$, we only keep the onset with the maximum predicted probability between $p b_{-} P(t P)$ and $p b_{-} S(t S)$. Here $d t_{-} S P \_n e a r[\mathrm{~s}]$ is an userdefined parameter. 

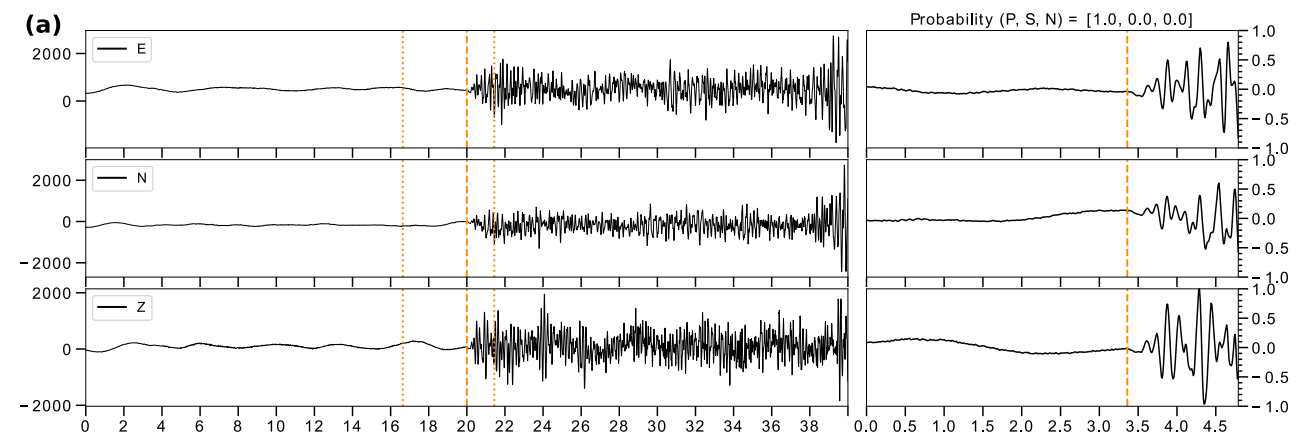

$$
\text { (b) }
$$
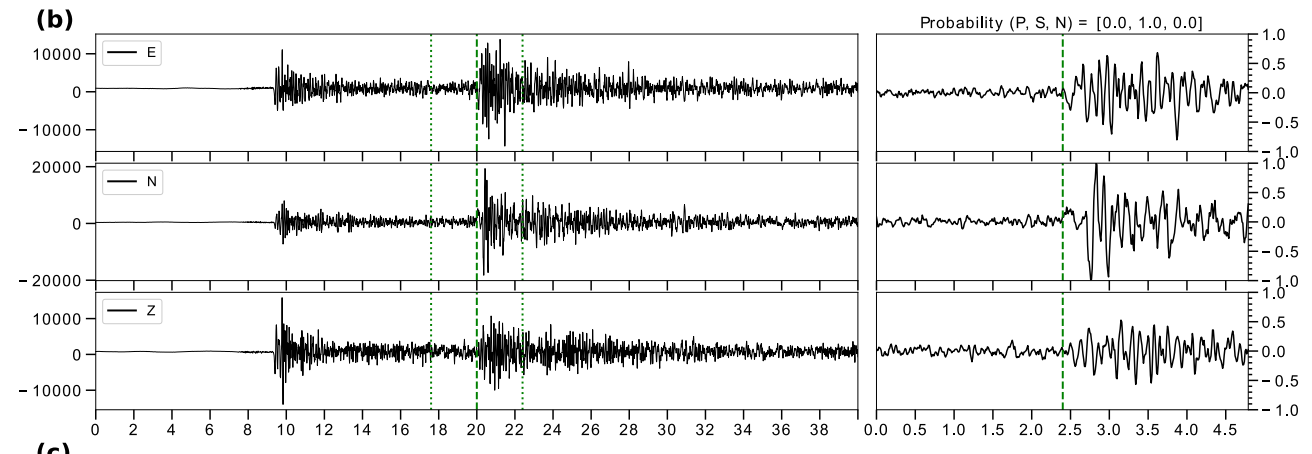

(c)
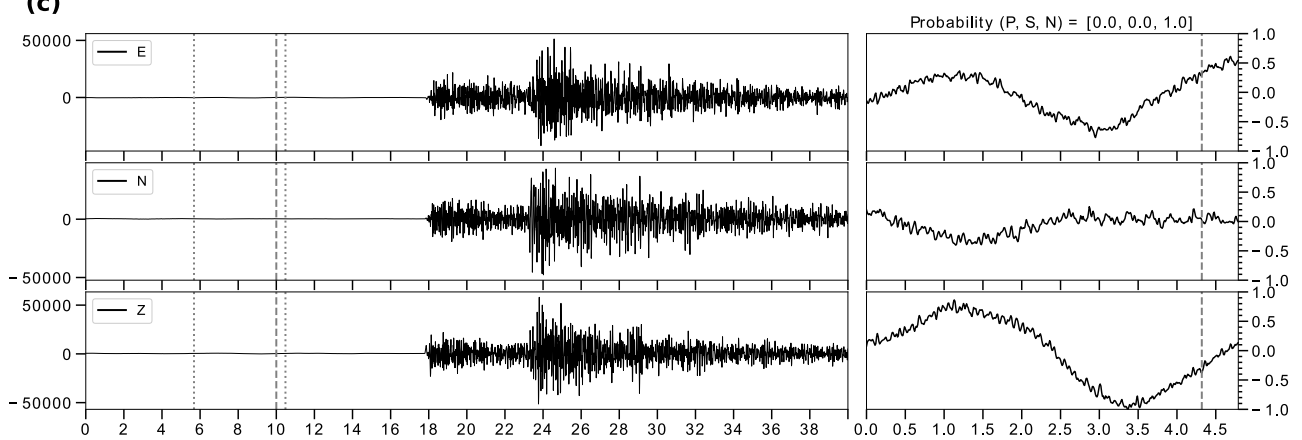

Figure S1. Examples of correctly predicted P- (a), S- (b) and N-class (c) samples in the test set of 4,320 samples. Subplots are plotted the same way as in Figure 8.
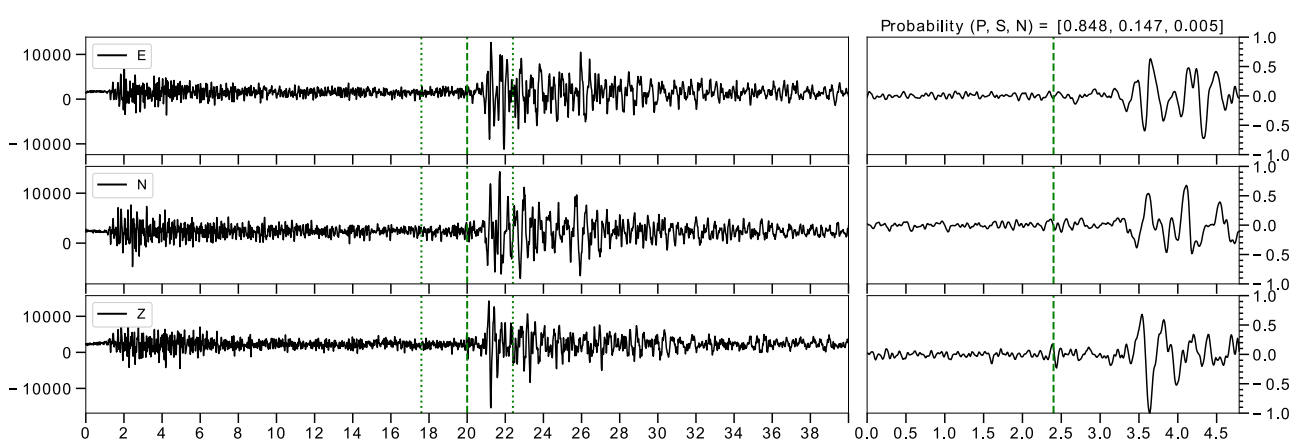

Figure S2. One example of $\mathrm{S}$ phase in the test set of 4,320 samples, which was misclassified by the network as $\mathrm{P}$ phase. Subplots are plotted the same way as in Figure 8. 

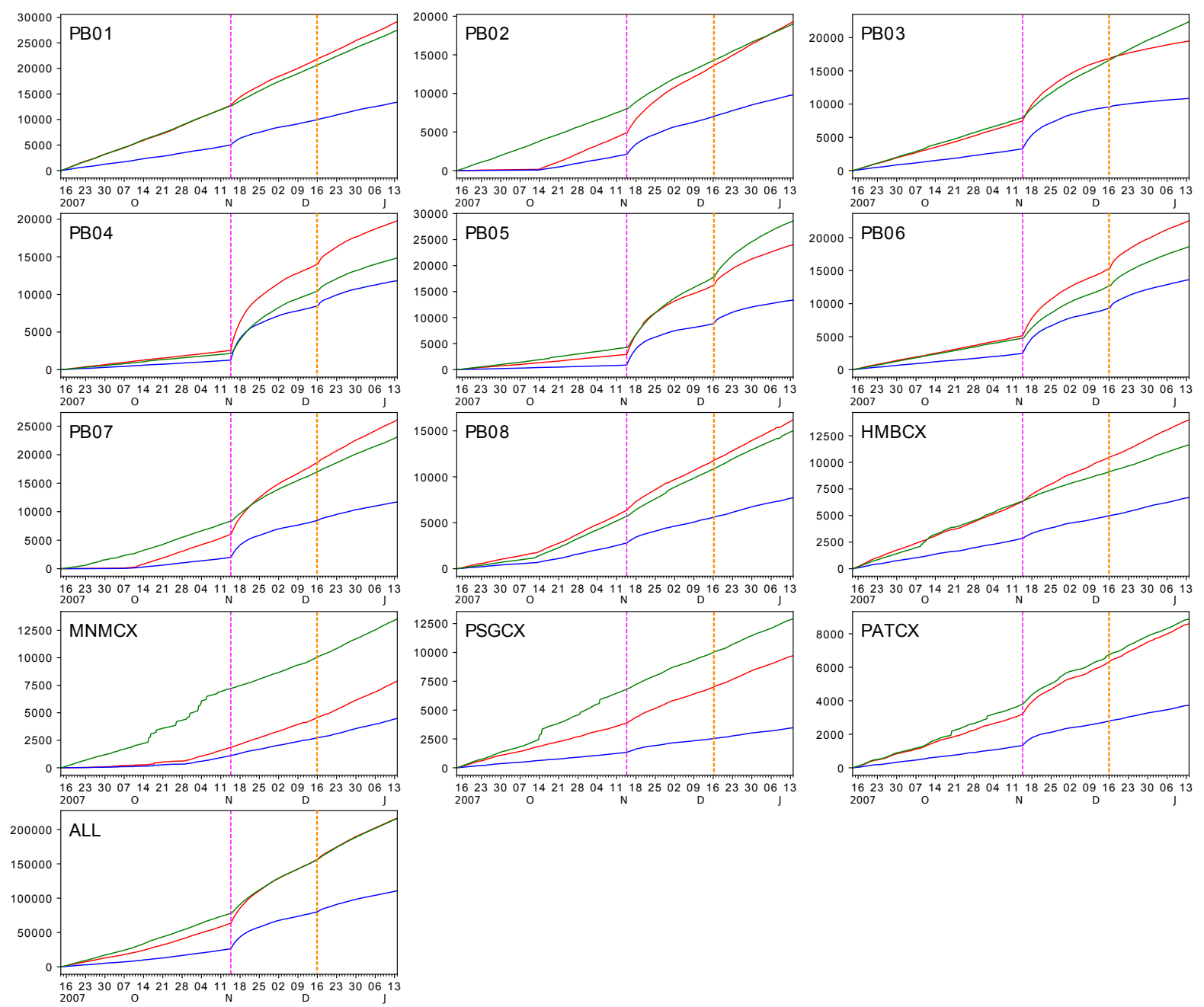

Figure S3. Cumulative number of P (red curve) and S (blue curve) phases predicted by DeepPhasePick $\left(p b_{-} P \_t h 1=p b_{-} S_{-} t h 1=0.98\right)$, and triggered detections by the STA/LTA algorithm (green curve) on continuous seismic data recorded by the stations with available data in northern Chile. Last subplot shows the combined results for all the stations in the network. Cumulative curves are plotted for the time interval between two months before and two months after the 2007 M7.7 Tocopilla mainshock (magenta dashed line). An orange dashed line indicates the time of occurrence of a M7.1 aftershock (2007-12-16) 50 km south of the mainshock epicenter. 

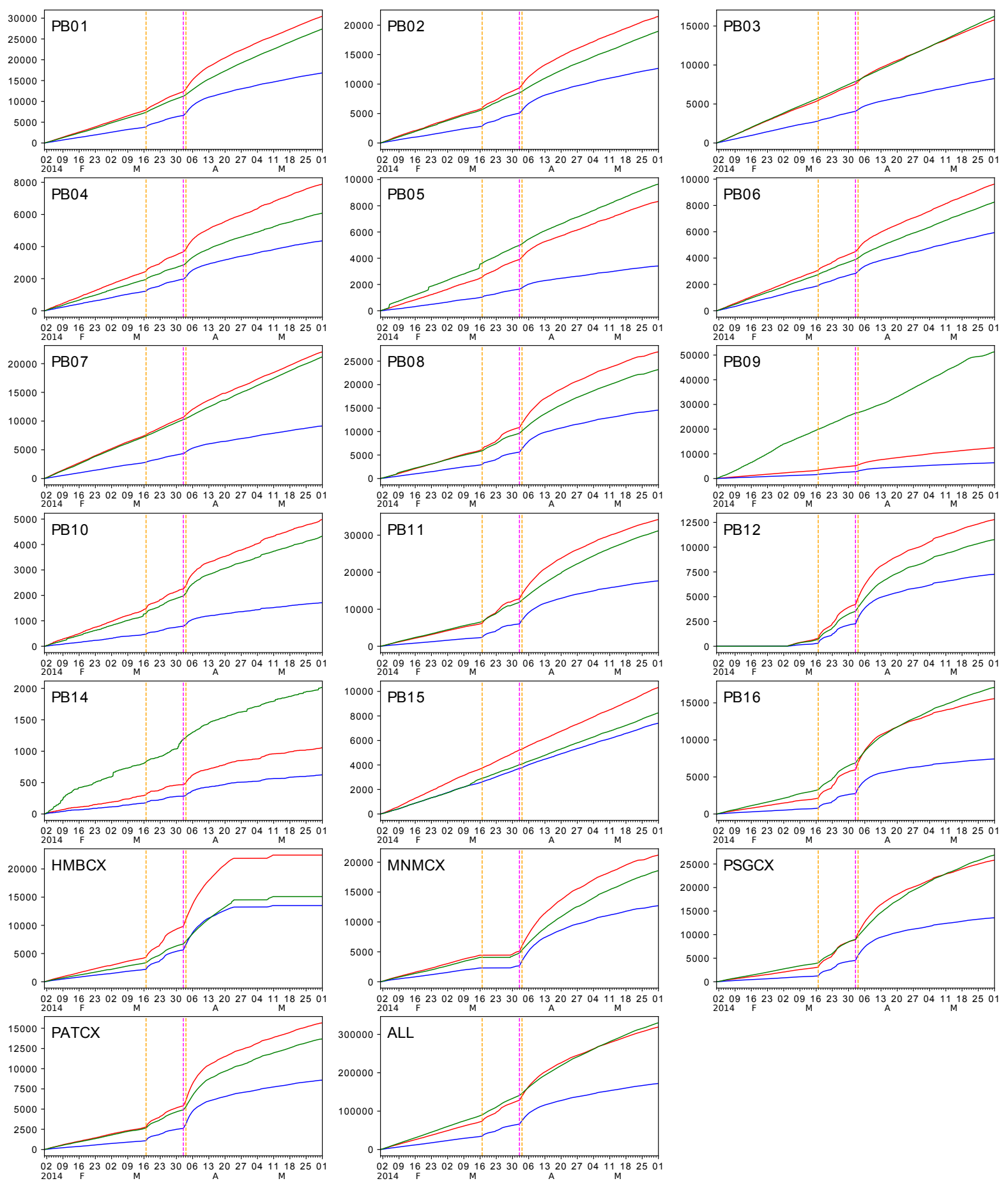

Figure S4. Cumulative number of P (red curve) and S (blue curve) phases predicted by DeepPhasePick $\left(p b \_P \_t h 1=p b \_S \_t h 1=0.98\right)$, and triggered detections by the STA/LTA algorithm (green curve) on continuous seismic data recorded by the stations with available data in northern Chile. Last subplot shows the combined results for all the stations in the network. Cumulative curves are plotted for the time interval between two months before and two months after the 2014 M8.1 Iquique mainshock (magenta dashed line). Two additional orange dashed lines indicate the time of occurrence of the M6.7 foreshock (2014-03-16) and the M7.6 aftershock (2014-04-03) in the Iquique sequence. 

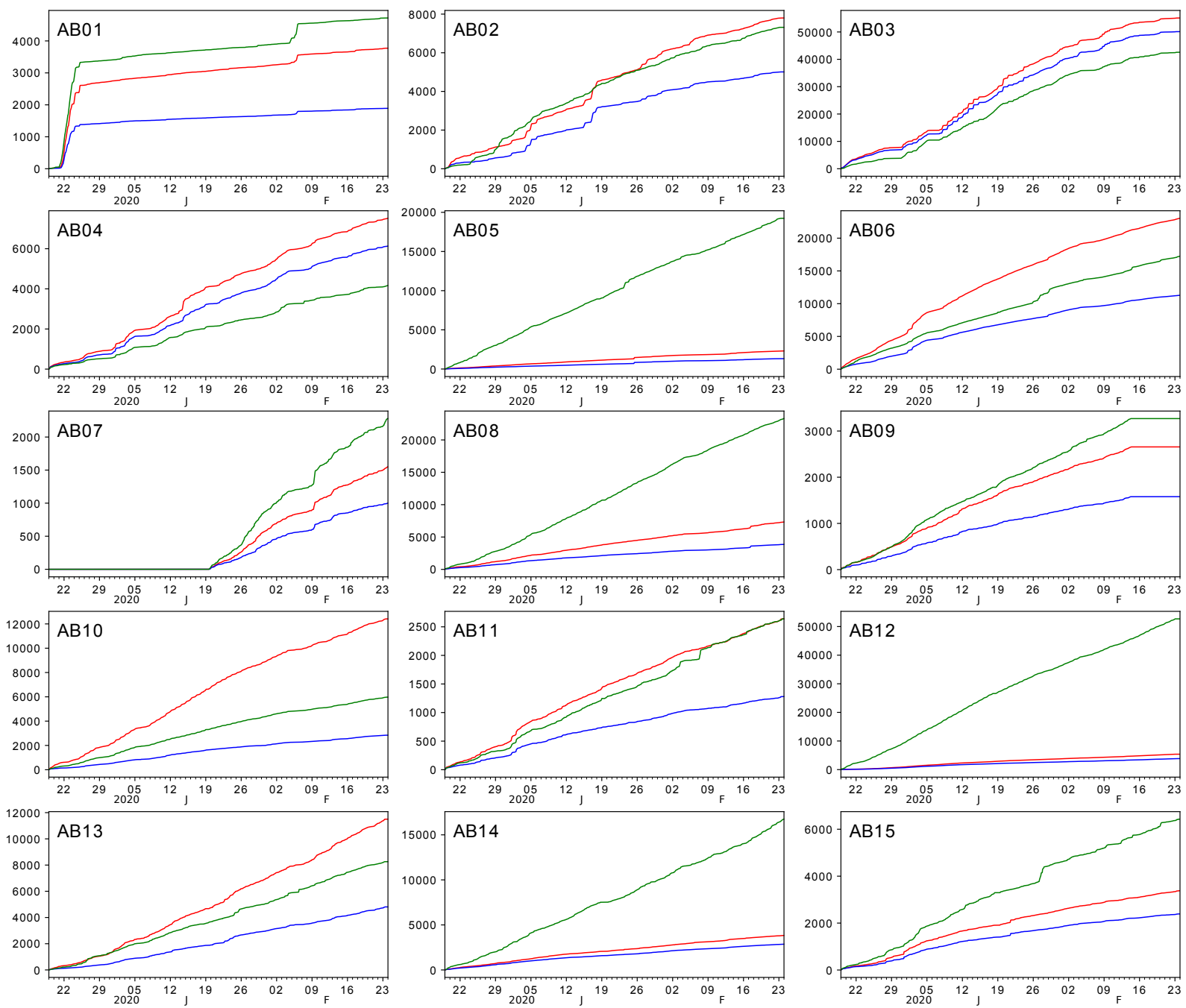

Figure S5. Cumulative number of $\mathrm{P}$ (red curve) and $\mathrm{S}$ (blue curve) phases predicted by DeepPhasePick $\left(p b_{-} P_{-} t h 1=p b_{-} S \_t h 1=0.98\right)$, and triggered detections by the STA/LTA algorithm (green curve) on continuous seismic data recorded by the stations with available data in Albania. Cumulative curves are plotted for the time interval between 2019-12-19 and 2020-02-24, during the aftershock sequence of the 2019 M6.4 Albania earthquake. First 15 stations in the network are shown here. 

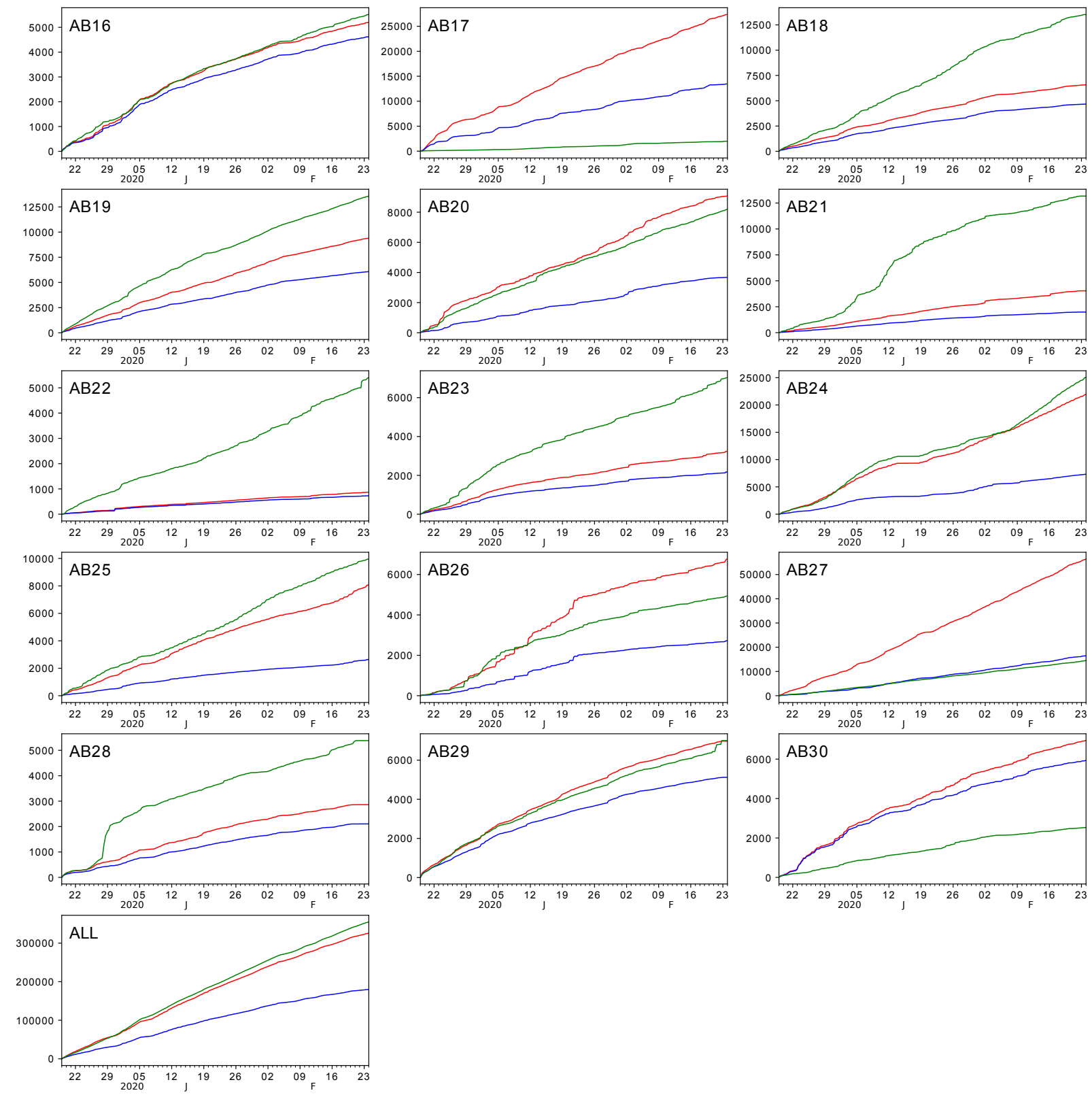

Figure S6. Similar to Figure S5, showing results from remaining stations in the Albanian network. Last subplot shows the combined results for all the stations in the network. 

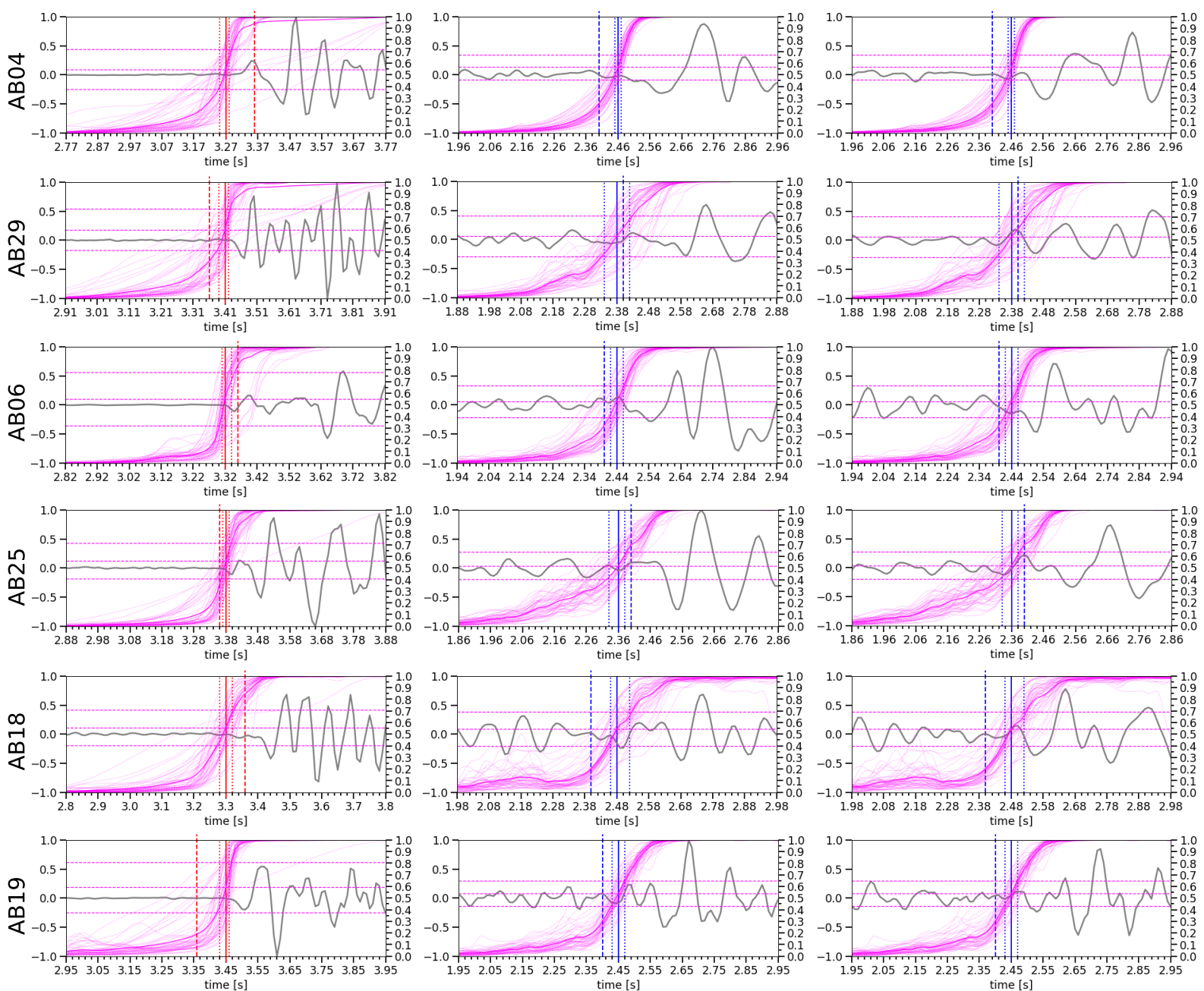

Figure S7. Similar to Figure 22, for detected event $\mathbf{e} 7$ in Figure 21. Pick statistics for this event are reported in Table $\mathbf{S 2}$. 

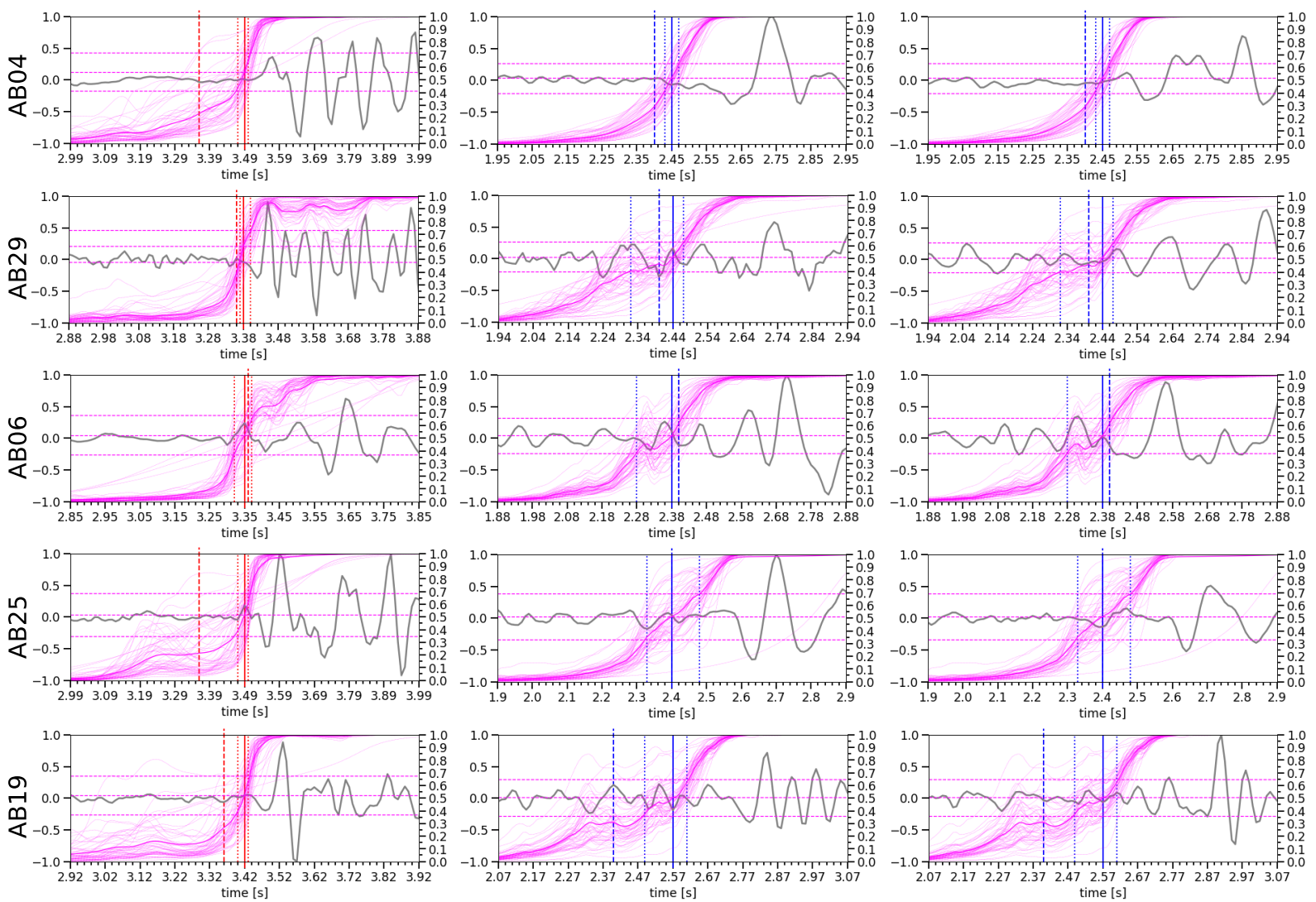

Figure S8. Similar to Figure 22, for detected event $\mathbf{e 8}$ in Figure 21. Pick statistics for this event are reported in Table $\mathrm{S3}$.

Table S1. Statistics of predicted picks shown in Figure 22 for event e1 in Figure 21.

\begin{tabular}{lccccccc}
\hline station & phase & tons_prelim $[\mathrm{s}]$ & tons_pred $[\mathrm{s}]$ & tons_err $(-,+)[\mathrm{s}]$ & pick_class & $p b$ & $p b_{\text {ststd }}$ \\
\hline $\mathrm{AB} 04$ & $\mathrm{P}$ & 3.36 & 3.41 & $(0.010,0.010)$ & 0 & 0.635 & 0.207 \\
$\mathrm{AB} 04$ & $\mathrm{~S}$ & 2.40 & 2.37 & $(0.010,0.010)$ & 0 & 0.523 & 0.114 \\
$\mathrm{AB} 29$ & $\mathrm{P}$ & 3.36 & 3.39 & $(0.010,0.010)$ & 0 & 0.611 & 0.198 \\
$\mathrm{AB} 29$ & $\mathrm{~S}$ & 2.40 & 2.43 & $(0.030,0.020)$ & 0 & 0.503 & 0.111 \\
$\mathrm{AB} 06$ & $\mathrm{P}$ & 3.36 & 3.30 & $(0.010,0.050)$ & 0 & 0.625 & 0.249 \\
$\mathrm{AB} 06$ & $\mathrm{~S}$ & 2.40 & 2.42 & $(0.040,0.030)$ & 0 & 0.536 & 0.175 \\
$\mathrm{AB} 25$ & $\mathrm{P}$ & 3.36 & 3.38 & $(0.040,0.040)$ & 0 & 0.507 & 0.253 \\
$\mathrm{AB} 25$ & $\mathrm{~S}$ & 2.40 & 2.42 & $(0.030,0.020)$ & 0 & 0.539 & 0.140 \\
$\mathrm{AB} 18$ & $\mathrm{P}$ & 3.36 & 3.38 & $(0.020,0.020)$ & 0 & 0.580 & 0.208 \\
$\mathrm{AB} 18$ & $\mathrm{~S}$ & 2.40 & 2.37 & $(0.060,0.020)$ & 0 & 0.500 & 0.117 \\
$\mathrm{AB} 19$ & $\mathrm{P}$ & 3.36 & 3.41 & $(0.030,0.020)$ & 0 & 0.511 & 0.215 \\
$\mathrm{AB} 19$ & $\mathrm{~S}$ & 2.40 & 2.41 & $(0.030,0.020)$ & 0 & 0.513 & 0.128 \\
\hline
\end{tabular}


Table S2. Statistics of predicted picks shown in Figure $\mathbf{S 7}$ for event e7 in Figure 21.

\begin{tabular}{lccccccc}
\hline station & phase & tons_prelim $[\mathrm{s}]$ & tons_pred $[\mathrm{s}]$ & tons_err $(-,+)[\mathrm{s}]$ & pick_class & $p b$ & pb_std \\
\hline $\mathrm{AB} 04$ & $\mathrm{P}$ & 3.36 & 3.27 & $(0.020,0.010)$ & 0 & 0.547 & 0.171 \\
$\mathrm{AB} 04$ & $\mathrm{~S}$ & 2.40 & 2.46 & $(0.010,0.010)$ & 0 & 0.564 & 0.109 \\
$\mathrm{AB} 29$ & $\mathrm{P}$ & 3.36 & 3.41 & $(0.020,0.010)$ & 0 & 0.591 & 0.176 \\
$\mathrm{AB} 29$ & $\mathrm{~S}$ & 2.40 & 2.38 & $(0.040,0.040)$ & 0 & 0.527 & 0.175 \\
$\mathrm{AB} 06$ & $\mathrm{P}$ & 3.36 & 3.32 & $(0.010,0.020)$ & 0 & 0.551 & 0.229 \\
$\mathrm{AB} 06$ & $\mathrm{~S}$ & 2.40 & 2.44 & $(0.020,0.020)$ & 0 & 0.529 & 0.139 \\
$\mathrm{AB} 25$ & $\mathrm{P}$ & 3.36 & 3.38 & $(0.010,0.010)$ & 0 & 0.561 & 0.152 \\
$\mathrm{AB} 25$ & $\mathrm{~S}$ & 2.40 & 2.36 & $(0.030,0.020)$ & 0 & 0.519 & 0.117 \\
$\mathrm{AB} 18$ & $\mathrm{P}$ & 3.36 & 3.30 & $(0.020,0.020)$ & 0 & 0.556 & 0.154 \\
$\mathrm{AB} 18$ & $\mathrm{~S}$ & 2.40 & 2.48 & $(0.020,0.040)$ & 0 & 0.544 & 0.148 \\
$\mathrm{AB} 19$ & $\mathrm{P}$ & 3.36 & 3.45 & $(0.020,0.010)$ & 0 & 0.592 & 0.218 \\
$\mathrm{AB} 19$ & $\mathrm{~S}$ & 2.40 & 2.45 & $(0.020,0.020)$ & 0 & 0.539 & 0.109 \\
\hline
\end{tabular}

Table S3. Statistics of predicted picks shown in Figure S8 for event e8 in Figure 21.

\begin{tabular}{lccccccc}
\hline station & phase & tons_prelim $[\mathrm{s}]$ & tons_pred $[\mathrm{s}]$ & tons_err $(-,+)[\mathrm{s}]$ & pick_class & $p b$ & pb_std \\
\hline $\mathrm{AB} 04$ & $\mathrm{P}$ & 3.36 & 3.49 & $(0.020,0.010)$ & 0 & 0.562 & 0.150 \\
$\mathrm{AB} 04$ & $\mathrm{~S}$ & 2.40 & 2.45 & $(0.020,0.020)$ & 0 & 0.515 & 0.120 \\
$\mathrm{AB} 29$ & $\mathrm{P}$ & 3.36 & 3.38 & $(0.010,0.020)$ & 0 & 0.604 & 0.127 \\
$\mathrm{AB} 29$ & $\mathrm{~S}$ & 2.40 & 2.44 & $(0.120,0.030)$ & 1 & 0.514 & 0.117 \\
$\mathrm{AB} 06$ & $\mathrm{P}$ & 3.36 & 3.35 & $(0.030,0.020)$ & 0 & 0.524 & 0.157 \\
$\mathrm{AB} 06$ & $\mathrm{~S}$ & 2.40 & 2.38 & $(0.100,0.020)$ & 1 & 0.521 & 0.138 \\
$\mathrm{AB} 25$ & $\mathrm{P}$ & 3.36 & 3.49 & $(0.020,0.010)$ & 0 & 0.518 & 0.172 \\
$\mathrm{AB} 25$ & $\mathrm{~S}$ & 2.40 & 2.40 & $(0.070,0.080)$ & 1 & 0.510 & 0.180 \\
$\mathrm{AB} 19$ & $\mathrm{P}$ & 3.36 & 3.42 & $(0.020,0.010)$ & 0 & 0.521 & 0.153 \\
$\mathrm{AB} 19$ & $\mathrm{~S}$ & 2.40 & 2.57 & $(0.080,0.040)$ & 1 & 0.506 & 0.145 \\
\hline
\end{tabular}

